\title{
Flora of Lake Seyfe Nature Reserve Area Kirsehir - Turkey
}

\author{
Omer Eyuboglu \\ Ahievran University, Faculty of Education, Kirsehir - Turkey \\ E.mail: oeyuboglu@ ahievran.edu.tr
}

\begin{abstract}
This research encompasses flora of Lake Seyfe (Kirsehir) Nature Reserve Area. 65 families, 237 genera, 383 species, 3 sub-species, and 2 varieties were determined with the evaluation of 874 plant specimens collected during 1993-1994 in the research area. The total taxon number was 388. 52 species were endemic for Turkey. The distribution and ratios of the species in the phytogeographic regions were as follows: 104 Iranian-Turanian components (21.75\%), 29 European-Siberian components (7.57\%), 13 Mediterranean components (3.39\%), 82 cosmopolitan components (21.40\%), and 155 components with unknown phytogeographic region $(40.46 \%)$.
\end{abstract}

Key Words: Lake Seyfe, flora, taxon.

DOI: $10.7176 / \mathrm{JSTR} / 5-2-05$

\section{INTRODUCTION}

Research on flora of Turkey started by the beginning of the 18th century with the travels of French botanist Tournefort in the Northern and Northeastern Anatolia during 1700-1702 and following it, some international botanists collected plants in Anatolia and its environs. The travel of Swedish botanist Boissier in Anatolia in 1842 was the start of research of Turkey's flora. Boissier's work titled "Flora Orientalis" is important as a major resource covering the plants of Turkey. Following Boissier, international researchers including Zohary, Sorger, Huber-Morartl1, and Mc Neill, and national researchers including Birand, Kasaplığil, Karamanoğlu, Akman, Yurdakulol, Ekim, and Ketenoğlu conducted studies on the flora and vegetation of Turkey.

The number of researches on flora of Turkey increased in this Century gradually and advanced substantially during the past 25-30 years. Especially, the work titled "Flora of Turkey and Eastern Aegean Islands" started to be published by P. H. Davis et al, in 1965 and completed as 10 volumes was the first important step taken on this path. Floristic studies in Turkey became important for Turkish botanists after starting flora publications, and studies in this field increased gradually. In parallel to floristic studies, vegetation studies increased during the past 25-30 years and the vegetation of specific areas was examined in terms of plant sociology and plant ecology, new plant colonies were defined and great steps were taken to discover vegetation of Turkey.

"Lake Seyfe Nature Reserve Area", which was selected as the research area, is in Kırşehir City limits and covers an area of 10,700 hectares. It was designated as the Nature Reserve Area on 28/6/1990 and was included in the list of the Convention on Wetlands of International Importance as per the decree of the Grand National Assembly of Turkey on 30/12/1993.

There is no detailed research conducted on the flora of the research area up until today. In the study of A. Çırpıcı titled "the areas studied in terms of flora and vegetation of Turkey", our study area was included in the group of areas never studied floristically. Lake Seyfe Nature Reserve Area is included in B5 square according to the grid system used in the work titled "Flora of Turkey" of Davis.

In this study, flora of Lake Seyfe Nature Reserve Area was determined. Hence, we believe that we have contributed to flora of Kırşehir City and Turkey. 


\section{GENERAL INFORMATION}

Lake Seyfe is located at 30km northeast of Kırşehir City, and $25 \mathrm{~km}$ North of Mucur County, and its altitude is $1110 \mathrm{~m}$. The lake and the area surrounding it is 10,700 of hectares. The area covered by the Lake ranges between 3,400 ha and 7,000 ha.

The mountainous areas surrounding Seyfe closed basin are not very high. The highest place near the research area is Kervansaray Mountain $(1,679 \mathrm{~m})$. The other important ridges are Kartaltepe $(1,665 \mathrm{~m})$, Armutlu Tepe (1,581 m) and Ayrıdağ $(1,550 \mathrm{~m})$.

Slightly waved prairies make up the Lake's environs. There are only a few streams located in the basin due to semi-arid climate. These streams are not continuous streams and the number of them increases with snow melting and increased rain fall during spring months. The streams diminish gradually and dry as a result of severe vaporization during summer months. The most critical water source supplying the Lake is the spring near Seyfe Village. In addition, the Lake is supplied from the bottom by underground water resources.

There are no fish living in the entire Lake in general. However, small fish species in the length of 5-6 cm including Aphanius chantra and Spirlinus sp. live in the places where the streams mix with the Lake. Despite these fish have no commercial value, they are important ecologically as the food of aquatic birds including pelicans and herns.

The Lake is special for its rich phytoplanktons and zooplanktons for feding of aquatic birds. Moreover, other species living in salty lake water are small shrimp-like crustacean (Artemia sp.) and some earthworms and fishworms. These species are the essential food sources of flamingos especially.

The most important species are aquatic birds, also called avifauna, living as dependent to the unique ecosystem formed by salty and alkaline water of Lake Seyfe.

Lake Seyfe is a reproduction, feeding and shelter environment for avifauna that is vital for not only Turkey but the world. 180 bird species were determined to exist in the Lake up until today. Considering that bird species are decreasing gradually and some species are becoming extinct due to destruction of ecologic systems throughout the world, the richness of bird species in Lake Seyfe becomes even more prominent.

Summers are warm and winters are cold in the research area. Depsite precipitation occurs in every season, it is in the form of snow during winter months in general and mostly. There was no considerable change seen in meteorologic features in the research area and its environs. The annual areal total precipitation amount was estimated as $353 \mathrm{~mm}$. Precipitation in the research area increased towards North. Annual precipitation amount was $373 \mathrm{~mm}$ according to the 56-year observations recorded in Kurşehir observation station, which is the closest station to Lake Seyfe.

Maximum amount of precipitation is received in January and minimum amount is received in July. There is no considerable difference between winter and spring months. In the other selected three stations, maximum precipitation is received in winter months, and in Lake Seyfe Basin, maximum precipitation is received in spring months. Climatic data on the research area were received from the archives of Ankara Meteorology Directorship and the Prime Ministry, General Directorate of Turksih State Meteorologic Service publications.

It was seen that the dominant wind direction was northeast based on the data collected at Kırşehir Meteorology Observation Station. Maximum monthly wind velocity ranges between $4-5 \mathrm{~m} / \mathrm{sec}$ in general. Maximum wind velocity value $(5 \mathrm{~m} / \mathrm{sec})$ occurs in July and minimum value $(3.5 \mathrm{~m} / \mathrm{sec})$ occurs in November. Wind velocity is lower in winter months generally in comparison to summer months. Minimum wind effect occurs in northwest-southeast direction. 


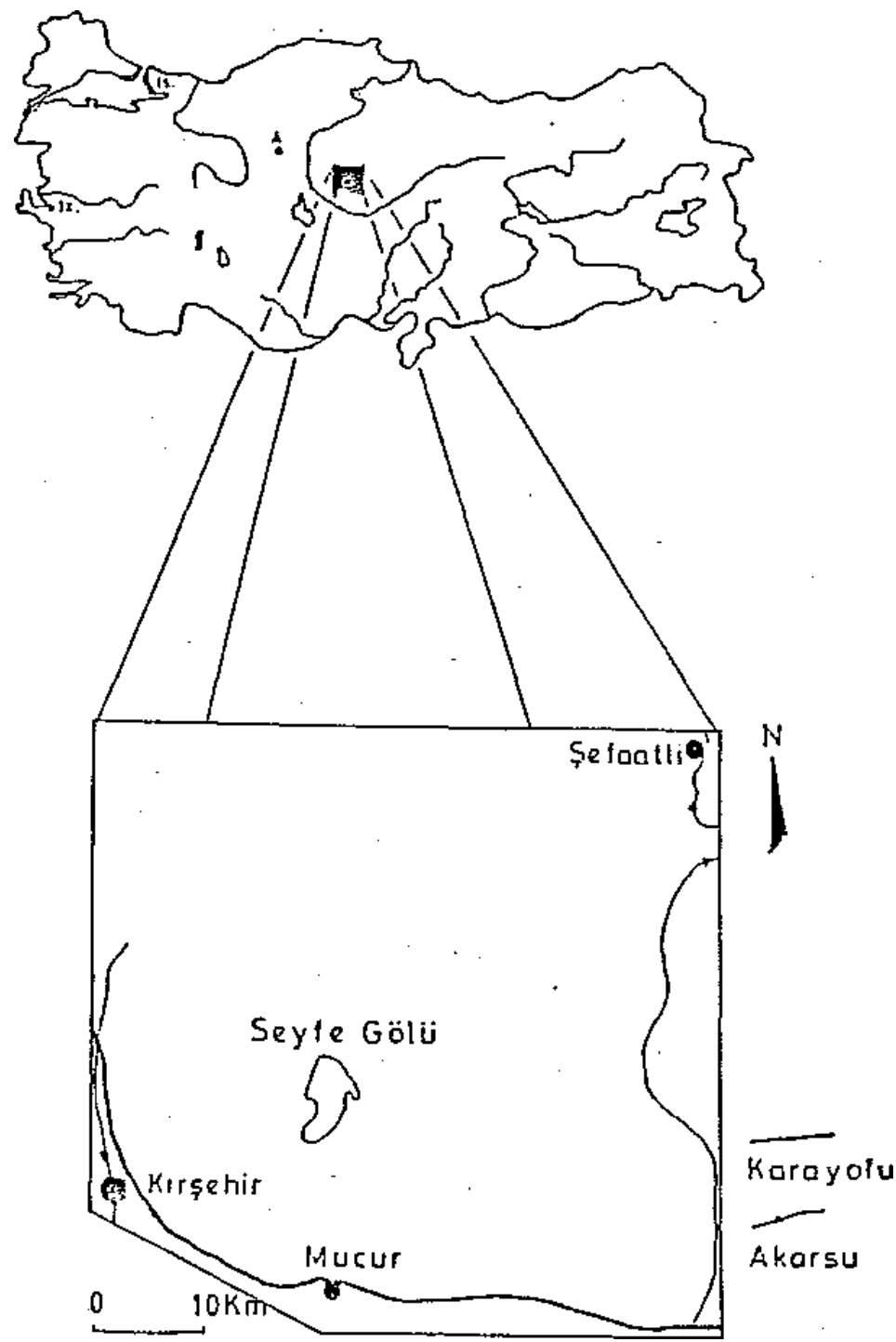

Map 1. Location Map of the Research Area

Lake Seyfe / karayolu: highway / akarsu: stream

\section{Research Area Vegetation}

The research area is situated in Iranian-Turanian region, one of the three phytogeographic regions of Turkey, and it is included in B5 square of the grid system. Lake Seyfe is located at 16km North of Kırşehir City, Mucur County. It is the most vital wetlands of Turkey. It appears like a bowl since it is surrounded by tall mountains and hills. It is a completely closed basin. This closed basin's area is more than $925 \mathrm{~km} 2$ and small springs and streams run to Lake Seyfe. Therefore, a lake of $35 \mathrm{~km} 2$ of area and a bog of $25 \mathrm{~km} 2$ of area are formed. The lake surface decreases and rises depending on precipitations. This plain is formed within a synclinal fold. This syncline was filled with volcanic and alluvial materials of 
hundreds of meters of thickness during geological periods. The emerging field has a salty character since the formation contains gypsium and excessive amounts of soluble salts and the salty lake covering this field retreated.

The Central Anatolian steppe vegetation gained a secondary appearance today with long-term anthropogenic effects. Our study area was destroyed substantially with the effect of factors including over grazing, land clearing, lake ebb and flow, and the borders of homogenous and characteristic pure steppe groups shrunk.

\section{1-Halophilic Vegetation}

In the research area, halophilic vegetation is represented by plant species including Halocnemum strobilaceum, Salicornia prostrata, Salsola inermis, Panderia pilosa, Petrosimonia brachiata, Krascheninnikovia ceratoides, Camphorosma monspeliaca; Gypsophila perfoliala, Frankenia hirsuta, Limonium iconicum, and Limonium globuliferum.

Halophilic plants are wooly and succulent plants resistant to low or high salt concentration and have dull colored herbaceous leaf type.

\section{2-Steppe Vegetation}

Lands owned by Malya Agricultural Enterprises Directorate made up a section of our research area. In a large section of the Enterprise, steppe vegetation was dominant. Plants composing this vegetation were thorny, cushion form xerophytes, xerophyte weeds, chamaephytes and hemicryptophytes.

Vegetation spread over on marn limestone or gypsium-bearing layers generally.

The most prevalent species forming the steppe vegetation in our area were as follows; Alyssum desertorum var. desertorum, A.pateri subsp. pateri, Spergularia media, Gypsophila parva, Noaea mucronata subsp. mucronata, Reaumaria atlernifolia, Astragalus kirshehiricus, A.lydius, A.karamasicus, Peganum harmala, Bupleurum sulphureum, Scabiosa argentea, Anthemis wiedemanniana, Achillea wilhelmsii, Artemisia santonicum, A.taurica, Gundelia tournefortii var. tournefortii, Centaurea corduiformis subsp. carduiformis, Lappula barbata, Bungea trifida, Phlomis armeniaca, Marrubium parviflorum subsp. oligodon, Thymus sipyleus subsp. rosulans, Acantholimon acerosum var. acerosum, Secale montanum, Bromus japonicus subsp. japonicus, B.tectorum, B.tomentellus, Festuca callieri subsp. callieri, and Stipa capillata.

\section{3-Praire Vegetation}

Our research area covers a wide wetland with no incline in Karaçayır locality. This area is full of abundant number of Juncaceae, Poaceae and Cyperaceae members. Some of these are Jucus gerardi subsp. gerardi, Eleocharis uniglumis, Bolboschoenus maritimus var. maritimus, Carex otrubae, C.divisa, Dactylis glomerata subsp. hispanica, Cynodon dactylon var. villosus, and Elymus elongatus subsp. ponticus.

Furthermore, it the other wide wetlands and meadows, the following species are prevalent; Triglochin palustris, Butomus umbellatus, Alisma plantogo- aquatica, Orcbis palustris, Typha latifolia, Phragmites australis, Aeluropus littoralis, Lotus corniculatus var. corniculatus, Tetragonolobus maritimus, and Glaux maritima species.

Eyüboğlu, Ö. published previously some sections of his doctoral dissertation study conducted in Lake Seyfe Basin in 1995 and titled "Flora of Lake Seyfe Nature Reserve Area, Kırşehir-Turkey". "New Floristic Records from Anatolia (B5)" was published in 1995; "Protection of Natural Life Chain of Lake Seyfe and its Vicinity" was published in 1996; and "Endemic Plants of Lake Seyfe Basin" was published in 1998 in various peer-reviewed journals. 
LIST OF LAKE SEYFE BASIN PLANTS - FLORA

\begin{tabular}{|c|c|c|c|c|c|c|}
\hline No & Family & Species & $\begin{array}{l}\text { EUNIS Habitat } \\
\text { code }\end{array}$ & Endemism & $\begin{array}{l}\text { Phytogeographical } \\
\text { Region }\end{array}$ & $\begin{array}{l}\text { Endangerment } \\
\text { statue }\end{array}$ \\
\hline & PTERIDOPHYTA & & & & & \\
\hline 1 & Equisetaceae & Equisetum ramosissima Desf. & E2.5 & - & - & $\mathrm{LC}$ \\
\hline & SPERMATOPHYTA & & & & & \\
\hline & GYMNOSPERMAE & & & & & \\
\hline 2 & Cupressaceae & Thuja orientalis L. & G3 & - & - & $\mathrm{LC}$ \\
\hline 3 & Pinaceae & Cedrus libani A. Rich. & G3 & & - & $\mathrm{LC}$ \\
\hline 4 & Pinaceae & Pinus nigra An1. & G3 & - & - & $\mathrm{LC}$ \\
\hline \multirow[t]{3}{*}{5} & Pinaceae & Pinus sylvestris L. & G3 & & - & $\mathrm{LC}$ \\
\hline & ANGIOSPERMAE & & & & & \\
\hline & DICOTYLEDONES & & & & & \\
\hline 6 & Acanthaceae & Acanthus hirsutus Boiss. & E1.2 & Endemik & Ir.-Tur. ele. & $\mathrm{LC}$ \\
\hline 7 & Aceraceae & Acer negundo L. & E4.4 & - & - & $\mathrm{LC}$ \\
\hline 8 & Amaranthaceae & Amaranthus albus L. & E4.4 & - & & $\mathrm{LC}$ \\
\hline 9 & Apiaceae & Bupleurum croceum Fenzl & E4.4 & - & Ir.-Tur. ele. & $\mathrm{LC}$ \\
\hline 10 & Apiaceae & $\begin{array}{l}\text { Bupleurum heldreichii Boiss. \& } \\
\text { Bal. }\end{array}$ & E4.4 & Endemik & Ir.-Tur. ele. & $\mathrm{LC}$ \\
\hline 11 & Apiaceae & $\begin{array}{l}\text { Bupleurum sulphureum Boiss. } \\
\& \text { Bal. }\end{array}$ & E4.4 & Endemik & Ir.-Tur. ele. & $\mathrm{LC}$ \\
\hline 12 & Apiaceae & Bupleurum turcicum Snogerup & E2.5 & Endemik & Ir.-Tur ele. & $\mathrm{LC}$ \\
\hline 13 & Apiaceae & Caucalis platycarpos $\mathrm{L}$. & 11.3 & - & - & $\mathrm{LC}$ \\
\hline 14 & Apiaceae & Conium maculatum $\mathrm{L}$. & E2.5 & - & & $\mathrm{LC}$ \\
\hline 15 & Apiaceae & Daucus carota $\mathrm{L}$. & 11.3 & - & - & $\mathrm{LC}$ \\
\hline 16 & Apiaceae & $\begin{array}{l}\text { Echinophora tenuifolia } \text { L. } \\
\text { s11bsp. sibthorpiana (Guss.) } \\
\text { Tutin }\end{array}$ & E4.4 & - & Ir.-Tur. ele. & $\mathrm{LC}$ \\
\hline 17 & Apiaceae & Eryngium bithynicum Boiss. & E2.5 & Endemik & Ir.-Tur ele. & $\mathrm{LC}$ \\
\hline 18 & Apiaceae & $\begin{array}{l}\text { Eryngium campestre L. var. } \\
\text { virens Link }\end{array}$ & E4.4 & - & - & $\mathrm{LC}$ \\
\hline 19 & Apiaceae & Eryngium glomeratum Lam. & E4.4 & - & - & $\mathrm{LC}$ \\
\hline 20 & Apiaceae & $\begin{array}{l}\text { Falcaria falcarioides (Bomm. } \\
\& \text { Wolff.) Wolff. }\end{array}$ & E4.4 & - & - & $\mathrm{LC}$ \\
\hline 21 & Apiaceae & Falcaria vulgaris Bernh. & E4.4 & - & - & $\mathrm{LC}$ \\
\hline 22 & Apiaceae & Ferula rigidula DC. & E4.4 & - & Ir.-Tur ele. & $\mathrm{LC}$ \\
\hline 23 & Apiaceae & $\begin{array}{l}\text { Prangos melicarpoides Boiss. } \\
\text { var. melicarpoides }\end{array}$ & H5.3 & Endemik & Ir.-Tur. ele. & $\mathrm{LC}$ \\
\hline 24 & Apiaceae & Turgenia latifolia (L.) Hoffm. & E4.4 & - & - & $\mathrm{LC}$ \\
\hline 25 & Asclepiadaceae & $\begin{array}{l}\text { Cynanchum acutum L. subsp. } \\
\text { Acutum }\end{array}$ & E4.4 & - & - & $\mathrm{LC}$ \\
\hline 26 & Asteraceae & $\begin{array}{l}\text { Achillect aleppica DC. subsp. } \\
\text { zederbaueri (Hayek) Hub.-Mor. }\end{array}$ & E4.4 & Endemik & Ir.-Tur. ele. & $\mathrm{LC}$ \\
\hline 27 & Asteraceae & Achillea biebersteini Afan. & 11.3 & - & Ir.-Tur. ele. & $\mathrm{LC}$ \\
\hline
\end{tabular}




\begin{tabular}{|c|c|c|c|c|c|c|}
\hline 28 & Asteraceae & Achillea setacea Waldst. \& Kit. & E1.2 & & Avr. -Sib. ele. & $\mathrm{LC}$ \\
\hline 29 & Asteraceae & Achillea wilhelmsii C. Koch & $\mathrm{E} 4.4$ & - & Ir.-Tur. ele. & $\mathrm{LC}$ \\
\hline 30 & Asteraceae & $\begin{array}{l}\text { Anthemis cretica L. subsp, } \\
\text { anatolica (Boiss.) Grierson }\end{array}$ & $\mathrm{E} 4.4$ & - & - & $\mathrm{LC}$ \\
\hline No & Family & Species & $\begin{array}{l}\text { EUNIS Habitat } \\
\text { code }\end{array}$ & Endemism & $\begin{array}{l}\text { Phytogeographical } \\
\text { Region }\end{array}$ & $\begin{array}{l}\text { Endangerment } \\
\text { statue }\end{array}$ \\
\hline 31 & Asteraceae & $\begin{array}{l}\text { Anthemis tinctoria L. var. } \\
\text { Tinctoria }\end{array}$ & E4.4 & - & - & $\mathrm{LC}$ \\
\hline 32 & Asteraceae & $\begin{array}{l}\text { Anthemis wiedemanniana Fisch. } \\
\text { \& Mey. }\end{array}$ & E4.4 & Endemik & - & $\mathrm{LC}$ \\
\hline 33 & Asteraceae & $\begin{array}{l}\text { Arctium minus (Hill) Bernh. } \\
\text { subsp. pubens (Babington) } \\
\text { Arenes }\end{array}$ & $\mathrm{El} .2$ & - & Avr. -Sib. ele. & $\mathrm{LC}$ \\
\hline 34 & Asteraceae & Artemisia santonicum L. & E4.4 & - & Avr'. -Sib. ele. & $\mathrm{LC}$ \\
\hline 35 & Asteraceae & Artemisia taurica Willd. & $\mathrm{E} 4.4$ & - & - & $\mathrm{LC}$ \\
\hline 36 & Asteraceae & $\begin{array}{l}\text { Carduus nutans L. subsp. } \\
\text { Nutans }\end{array}$ & El.2 & - & - & $\mathrm{LC}$ \\
\hline 37 & Asteraceae & $\begin{array}{l}\text { Centaurea carduiformis DC. } \\
\text { subsp. carduiformis var. } \\
\text { Caduiformis }\end{array}$ & $\mathrm{E} 4.4$ & - & - & $\mathrm{LC}$ \\
\hline 38 & Asteraceae & Centaurea glastifolia L. & E4.4 & - & Ir.-Tur. ele. & $\mathrm{LC}$ \\
\hline 39 & Asteraceae & Centaurea iberica Trev. & $\mathrm{E} 1.2$ & - & - & $\mathrm{LC}$ \\
\hline 40 & Asteraceae & Centaurea patula DC. & $\mathrm{E} 4.4$ & - & Ir.-Tur. ele. & $\mathrm{LC}$ \\
\hline 41 & Asteraceae & $\begin{array}{l}\text { Centaurea picheri Boiss. subsp. } \\
\text { extrarosularis (Hayek \& Siehe) } \\
\text { Wagenitz }\end{array}$ & $\mathrm{E} 4.4$ & - & - & $\mathrm{LC}$ \\
\hline 42 & Asteraceae & $\begin{array}{l}\text { Centaurea solstitialis L. subsp. } \\
\text { Solstitialis }\end{array}$ & El.2 & - & - & $\mathrm{LC}$ \\
\hline 43 & Asteraceae & $\begin{array}{l}\text { Centaurea urvillei DC. subsp. } \\
\text { stepposa Wagenitz }\end{array}$ & $\mathrm{E} 4.4$ & & Ir.-Tur. ele. & $\mathrm{LC}$ \\
\hline 44 & Asteraceae & Centaurea virgata Lam. & $\mathrm{El} .2$ & - & - & 'LC \\
\hline 45 & Asteraceae & $\begin{array}{l}\text { Chardinia orientalis (L.) } \\
\text { O.Kuntze }\end{array}$ & $\mathrm{E} 4.4$ & - & Ir.-Tur. ele. & $\mathrm{LC}$ \\
\hline 46 & Asteraceae & Cichorium intybus L. & El .2 & - & - & $\mathrm{LC}$ \\
\hline 47 & Asteraceae & $\begin{array}{l}\text { Cirsium creticum (Lam.) d'Urv. } \\
\text { subsp. Creticum }\end{array}$ & $\mathrm{El} .2$ & - & Akd. ele. & $\mathrm{LC}$ \\
\hline 48 & Asteraceae & $\begin{array}{l}\text { Cirsium lappaceum (Bieb.) } \\
\text { Fischer subsp. anatolicum } \\
\text { Petrak }\end{array}$ & 11.3 & - & Ir.-Tur. ele. & $\mathrm{LC}$ \\
\hline 49 & Asteraceae & Cirsium vulgare (Savi) Ten. & $\mathrm{E} 1.2$ & - & - & $\mathrm{LC}$ \\
\hline 50 & Asteraceae & Cisium arvense (L.) Scop. & 11.3 & - & - & $\mathrm{LC}$ \\
\hline 51 & Asteraceae & Cousinia halyensis Hub.-Mor. & $\mathrm{E} 4.4$ & Endemik & Ir.-Tur. ele. & $\mathrm{LC}$ \\
\hline 52 & Asteraceae & $\begin{array}{l}\text { Crepis foetida } \text { L. subsp. } \\
\text { rhoeadifola (Bieb.) Celak. }\end{array}$ & H3.6 & - & - & $\mathrm{LC}$ \\
\hline 53 & Asteraceae & $\begin{array}{l}\text { Crepis macropus Boiss. \& } \\
\text { Heldr. }\end{array}$ & $\mathrm{E} 4.4$ & Endemik & Ir.-Tur. ele. & $\mathrm{LC}$ \\
\hline 54 & Asteraceae & $\begin{array}{l}\text { Crupina crupinastrum (Moris) } \\
\text { Vis. }\end{array}$ & $\mathrm{E} 4.4$ & - & - & $\mathrm{LC}$ \\
\hline 55 & Asteraceae & Echinops ritro L. & $\mathrm{E} 4.4$ & - & - & $\mathrm{LC}$ \\
\hline 56 & Asteraceae & $\begin{array}{l}\text { Gundelia tournefortii L. var. } \\
\text { Tournefortii }\end{array}$ & $\mathrm{E} 4.4$ & - & - & $\mathrm{LC}$ \\
\hline 57 & Asteraceae & Helianthus annuus L. & 11.3 & - & - & $\mathrm{LC}$ \\
\hline 58 & Asteraceae & $\begin{array}{l}\text { Helichrysum plicatum DC. } \\
\text { subsp. Plicaum }\end{array}$ & $\mathrm{E} 4.4$ & - & - & $\mathrm{LC}$ \\
\hline
\end{tabular}




\begin{tabular}{|c|c|c|c|c|c|c|}
\hline 59 & Asteraceae & $\begin{array}{l}\text { Helichrysum arenarium (L.) } \\
\text { Moench subsp. aucheri (Boiss.) } \\
\text { Davis \& Kupicha }\end{array}$ & $\mathrm{E} 4.4$ & Endemik & Ir.-Tur. ele. & $\mathrm{LC}$ \\
\hline 60 & Asteraceae & Inula oculus-christi L. & E4.4 & - & Avr. -Sib. ele. & $\mathrm{LC}$ \\
\hline No & Family & Species & $\begin{array}{l}\text { EUNIS Habitat } \\
\text { code }\end{array}$ & Endemism & $\begin{array}{l}\text { Phytogeographical } \\
\text { Region }\end{array}$ & $\begin{array}{l}\text { Endangerment } \\
\text { statue }\end{array}$ \\
\hline 61 & Asteraceae & Lactuca serriola $\mathrm{L}$. & 11.3 & - & Avr. -Sib. ele. & $\mathrm{LC}$ \\
\hline 62 & Asteraceae & $\begin{array}{l}\text { Leotodon asperrimus (Willd.) } \\
\text { J.Ball. }\end{array}$ & $\mathrm{E} 1.2$ & - & Ir.-Tur. ele. & $\mathrm{LC}$ \\
\hline 63 & Asteraceae & Logfia arvensis (L.) Holub & $\mathrm{E} 4.4$ & - & - & $\mathrm{LC}$ \\
\hline 64 & Asteraceae & $\begin{array}{l}\text { Onopordum analolicum (Boiss.) } \\
\text { Eig }\end{array}$ & $\mathrm{E} 4.4$ & Endemik & Ir.-Tur. ele. & $\mathrm{LC}$ \\
\hline 65 & Asteraceae & $\begin{array}{l}\text { Pilosella xcntviculoides } \\
\text { (A.F.Lang) Sell \& West }\end{array}$ & $\mathrm{E} 1.2$ & - & Ir.-Tur. ele. & $\mathrm{LC}$ \\
\hline 66 & Asteraceae & $\begin{array}{l}\text { Scariola ovientalis (Boiss.) } \\
\text { Sojak }\end{array}$ & $\mathrm{E} 1.2$ & - & Ir.-Tur. ele. & $\mathrm{LC}$ \\
\hline 67 & Asteraceae & Scolymus hispanicus L. & $\mathrm{E} 4.4$ & - & Akd. ele. & $\mathrm{LC}$ \\
\hline 68 & Asteraceae & Scorzonera parviflora Jacq. & El.2 & - & - & $\mathrm{LC}$ \\
\hline 69 & Asteraceae & Senecio vernalis Waldst. \& Kit. & $\mathrm{E} 4.4$ & - & - & $\mathrm{LC}$ \\
\hline 70 & Asteraceae & Taraxacum buttleri Van Soest & $\mathrm{E} 1.2$ & - & - & $\mathrm{LC}$ \\
\hline 71 & Asteraceae & $\begin{array}{l}\text { Taraxacum jerotinum (Waldst. } \\
\text { \& Kit.) Poiret }\end{array}$ & $\mathrm{E} 1.2$ & - & - & $\mathrm{LC}$ \\
\hline 72 & Asteraceae & $\begin{array}{l}\text { Tragopogon latifolius Boiss. } \\
\text { var. angustifolius Boiss. }\end{array}$ & El.2 & - & Ir.-Tur. ele. & $\mathrm{LC}$ \\
\hline 73 & Asteraceae & $\begin{array}{l}\text { Tripleurospermum decipiens } \\
\text { (Fisch. \& Mey) Bornm. }\end{array}$ & $\mathrm{E} 1.2$ & - & - & $\mathrm{LC}$ \\
\hline 74 & Asteraceae & $\begin{array}{l}\text { Tripleurospermum oreades } \\
\text { (Boiss.) Rech. var. oreades }\end{array}$ & $\mathrm{E} 2.5$ & - & - & $\mathrm{LC}$ \\
\hline 75 & Asteraceae & Xanthium spinosum L. & $\mathrm{E} 4.4$ & - & - & $\mathrm{LC}$ \\
\hline 76 & Asteraceae & Xeranthemum annuum $\mathrm{L}$. & $\mathrm{E} 4.4$ & - & - & $\mathrm{LC}$ \\
\hline 77 & Boraginaceae & $\begin{array}{l}\text { Alkanna orientalis (L.) Boiss. } \\
\text { var. Orientalis }\end{array}$ & $\mathrm{E} 4.4$ & - & Ir.-Tur. ele. & $\mathrm{LC}$ \\
\hline 78 & Boraginaceae & $\begin{array}{l}\text { Anchusa leptophylla Roemer \& } \\
\text { Schultes subsp. Leptophylla }\end{array}$ & $\mathrm{E} 1.2$ & - & - & $\mathrm{LC}$ \\
\hline 79 & Boraginaceae & $\begin{array}{l}\text { Cerinthe minör L. subsp. } \\
\text { auriculata (Ten.) Domac }\end{array}$ & $\mathrm{E} 1.2$ & - & - & $\mathrm{LC}$ \\
\hline 80 & Boraginaceae & Echium italicum L. & $\mathrm{E} 4.4$ & - & Akd. ele. & $\mathrm{LC}$ \\
\hline 81 & Boraginaceae & $\begin{array}{l}\text { Heliotropium lasiocarpum } \\
\text { Fisch. \& Mey. }\end{array}$ & $\mathrm{E} 4.4$ & - & Ir.-Tur. ele. & $\mathrm{LC}$ \\
\hline 82 & Boraginaceae & Lappula barbata (Bieb.) Gürke & $\mathrm{E} 4.4$ & - & Ir.-Tur. ele. & $\mathrm{LC}$ \\
\hline 83 & Boraginaceae & $\begin{array}{l}\text { Lappula squarrosa (Retz.) } \\
\text { Dumort }\end{array}$ & 11.3 & - & - & $\mathrm{LC}$ \\
\hline 84 & Boraginaceae & $\begin{array}{l}\text { Moltkia coerulea (Willd.) } \\
\text { Lehm. }\end{array}$ & $\mathrm{E} 4.4$ & - & Ir.-Tur. ele. & $\mathrm{LC}$ \\
\hline 85 & Boraginaceae & Onosma annemim DC. & E4.4 & Endemik & Ir.-Tur. ele. & $\mathrm{LC}$ \\
\hline 86 & Boraginaceae & $\begin{array}{l}\text { Onosma tauricum Pallas ex } \\
\text { Willd. var. Tauricum }\end{array}$ & $\mathrm{E} 4.4$ & Endemik & - & $\mathrm{LC}$ \\
\hline 87 & Brassicaceae & $\begin{array}{l}\text { Alyssum desertorum Stapf. var. } \\
\text { Desertorum }\end{array}$ & $\mathrm{E} 4.4$ & - & $-\bullet$ & $\mathrm{LC}$ \\
\hline 88 & Brassicaceae & $\begin{array}{l}\text { Alyssum pateri Nyar. subsp. } \\
\text { Pateri }\end{array}$ & $\mathrm{E} 4.4$ & Endemik & Ir.-Tur. ele. & $\mathrm{LC}$ \\
\hline 89 & Brassicaceae & $\begin{array}{l}\text { Alyssum praecox Boiss. \& Bal. } \\
\text { var, albiflorum Dudley }\end{array}$ & $\mathrm{E} 4.4$ & Endemik & Ir.-Tur. ele. & $\mathrm{LC}$ \\
\hline 90 & Brassicaceae & Alyssum sibiricum Willd. & H5.3 & - & - - & $\mathrm{LC}$ \\
\hline
\end{tabular}




\begin{tabular}{|c|c|c|c|c|c|c|}
\hline 91 & Brassicaceae & Arabis nova Vill. & $\mathrm{E} 4.4$ & - & - & $\mathrm{LC}$ \\
\hline 92 & Brassicaceae & Camelina rumelica Vel. & $\mathrm{E} 4.4$ & - & - & $\mathrm{LC}$ \\
\hline 93 & Brassicaceae & $\begin{array}{l}\text { Capsella bursa-pastoris (L.) } \\
\text { Medik, }\end{array}$ & $\mathrm{E} 4.4$ & - & - & $\mathrm{LC}$ \\
\hline No & Family & Species & $\begin{array}{l}\text { EUNIS Habitat } \\
\text { code }\end{array}$ & Endemism & $\begin{array}{l}\text { Phytogeographical } \\
\text { Region }\end{array}$ & $\begin{array}{l}\text { Endangerment } \\
\text { statue }\end{array}$ \\
\hline 94 & Brassicaceae & $\begin{array}{l}\text { Cardaria draba (L.) Desv. } \\
\text { subsp. chcalepensis (L.) } \\
\text { O.E.Schulz }\end{array}$ & $\mathrm{E} 4.4$ & - & - & LC \\
\hline 95 & Brassicaceae & $\begin{array}{l}\text { Cardaria draba (L.) Desv. } \\
\text { subsp. draba }\end{array}$ & $\mathrm{E} 4.4$ & - & - & $\mathrm{LC}$ \\
\hline 96 & Brassicaceae & $\begin{array}{l}\text { Descurainia sophia (L.) Webb } \\
\text { ex Prantl }\end{array}$ & $\mathrm{E} 2.5$ & - & - & $\mathrm{LC}$ \\
\hline 97 & Brassicaceae & $\begin{array}{l}\text { Erophila verna (L.) Chevall. } \\
\text { subsp. verna }\end{array}$ & E4.4 & - & - & $\mathrm{LC}$ \\
\hline 98 & Brassicaceae & $\begin{array}{l}\text { Erysimum crassipes Fisch. \& } \\
\text { Mey. }\end{array}$ & H5.3 & - & - & LC \\
\hline 99 & Brassicaceae & Erysimum torulosum Hub.-Mor. & $\mathrm{E} 4.4$ & Endemik & - & $\mathrm{LC}$ \\
\hline 100 & Brassicaceae & $\begin{array}{l}\text { Lepidium cartilagineum } \\
\text { (J.May.)Thell. }\end{array}$ & E2.5 & - & - & LC \\
\hline 101 & Brassicaceae & Lepidium latifolium L. & $\mathrm{E} 4.4$ & - & - & $\mathrm{LC}$ \\
\hline 102 & Brassicaceae & Malcolmia africana (L.) R.Br. & $\mathrm{E} 4.4$ & - & - & $\mathrm{LC}$ \\
\hline 103 & Brassicaceae & Rapistrum rugosum (L.) All. & 11.3 & - & - & $\mathrm{LC}$ \\
\hline 104 & Brassicaceae & Sinapis arvensis $\mathrm{L}$. & $\mathrm{E} 4.4$ & - & - & LC \\
\hline 105 & Brassicaceae & Sisymbrium altissimum $\mathrm{L}$. & E4.4 & - & - & $\mathrm{LC}$ \\
\hline 106 & Brassicaceae & Thlaspi perfoliatum L. & E4.4 & - & - & $\mathrm{LC}$ \\
\hline 107 & Campanulaceae & $\begin{array}{l}\text { Asyneuma rigidum (Willd.) } \\
\text { Grossh. subsp. rigidum }\end{array}$ & E4.4 & - & Ir.-Tur. ele. & $\mathrm{LC}$ \\
\hline 108 & Caryophyllaceae & $\begin{array}{l}\text { Arnaria ledebouriana Fenzl var. } \\
\text { ledebouriana }\end{array}$ & H5.3 & Endemik & - & $\mathrm{LC}$ \\
\hline 109 & Caryophyllaceae & $\begin{array}{l}\text { Cerastium dichotomam } \mathrm{L} . \\
\text { subsp. dichotomum }\end{array}$ & 11.3 & - & - & $\mathrm{LC}$ \\
\hline 110 & Caryophyllaceae & Dianthus anatolicus Boiss. & $\mathrm{E} 4.4$ & Endemik & - & $\mathrm{LC}$ \\
\hline 111 & Caryophyllaceae & Dianthus balansae Boiss. & $\mathrm{E} 4.4$ & Endemik & - & $\mathrm{LC}$ \\
\hline 112 & Caryophyllaceae & $\begin{array}{l}\text { Dianthus crinitus } \text { Sm. var. } \\
\text { crinitus }\end{array}$ & E4.4 & - & - & $\mathrm{LC}$ \\
\hline 113 & Caryophyllaceae & $\begin{array}{l}\text { Dianthus crinitus } \mathrm{Sm} \text {. var. } \\
\text { crossosepatalus Boiss. }\end{array}$ & H5.3 & & - & $\mathrm{LC}$ \\
\hline 114 & Caryophyllaceae & $\begin{array}{l}\text { Dianthus zonatus Fenzl var. } \\
\text { zonatus }\end{array}$ & $\mathrm{E} 4.4$ & - & - & $\mathrm{LC}$ \\
\hline 115 & Caryophyllaceae & Gysophila eriocalyx Boiss. & $\mathrm{E} 4.4$ & Endemik & Ir.-Tur. ele. & $\mathrm{LC}$ \\
\hline 116 & Caryophyllaceae & Gysophila parva Bark. & E4.4 & Endemik & Ir.-Tur. ele. & $\mathrm{LC}$ \\
\hline 117 & Caryophyllaceae & Gysophila perfoliata $\mathrm{L}$. & D6.1 & - & - & $\mathrm{LC}$ \\
\hline 118 & Caryophyllaceae & Gysophila pilosa Hudson & E4.4 & - & Ir.-Tur. ele. & LC \\
\hline 119 & Caryophyllaceae & $\begin{array}{l}\text { Minuartia anatolica (Boiss.) } \\
\text { Woron. var. arachnoidea } \mathrm{Mc} \\
\text { Neill. }\end{array}$ & E4.4 & Endemik & Ir.-Tur. ele. & $\mathrm{LC}$ \\
\hline 120 & Caryophyllaceae & $\begin{array}{l}\text { Minuartia erythrosepala } \\
\text { (Boiss.) Hand.-Mazz. var. } \\
\text { erythrosepala }\end{array}$ & H5.3 & - & - & $\mathrm{LC}$ \\
\hline 121 & Caryophyllaceae & $\begin{array}{l}\text { Minuartia hamata (Hausskn.) } \\
\text { Mattf. }\end{array}$ & E4.4 & - & - & LC \\
\hline
\end{tabular}




\begin{tabular}{|c|c|c|c|c|c|c|}
\hline 122 & Caryophyllaceae & $\begin{array}{l}\text { Minuartia hybrida (Vill.) } \\
\text { Schschk. subsp. turcica Mc } \\
\text { Neill }\end{array}$ & $\mathrm{E} 4.4$ & - & - & $\mathrm{LC}$ \\
\hline 123 & Caryophyllaceae & $\begin{array}{l}\text { Saponaria prostrata Willd. } \\
\text { subsp. prostrata }\end{array}$ & 11.3 & Endemik & Ir.-Tur. ele. & $\mathrm{LC}$ \\
\hline No & Family & Species & $\begin{array}{l}\text { EUNIS Habitat } \\
\text { code }\end{array}$ & Endemism & $\begin{array}{l}\text { Phytogeographical } \\
\text { Region }\end{array}$ & $\begin{array}{l}\text { Endangerment } \\
\text { statue }\end{array}$ \\
\hline 124 & Caryophyllaceae & $\begin{array}{l}\text { Silene dichotoma Ehrh. subsp. } \\
\text { dichotoma }\end{array}$ & E4.4 & - & - & $\mathrm{LC}$ \\
\hline 125 & Caryophyllaceae & Silene marschallii C.A.Meyer & H3.6 & - & Ir.-Tur. ele. & $\mathrm{LC}$ \\
\hline 126 & Caryophyllaceae & $\begin{array}{l}\text { Silene spergulifolia (Desf.) } \\
\text { Bieb. }\end{array}$ & H3.6 & - & Ir.-Tur. ele. & $\mathrm{LC}$ \\
\hline 127 & Caryophyllaceae & $\begin{array}{l}\text { Silene vulgaris (Moench) } \\
\text { Garcke var. vulgaris }\end{array}$ & G3 & - & - & $\mathrm{LC}$ \\
\hline 128 & Caryophyllaceae & Spergularia media (L.) C.Presl & $\mathrm{E} 2.5$ & - & - & $\mathrm{LC}$ \\
\hline 129 & Chenopodiaceae & Atriplex laevis C.A.Meyer & $\mathrm{E} 2.5$ & - & - & $\mathrm{LC}$ \\
\hline 130 & Chenopodiaceae & Atriplex rosea $\mathrm{L}$. & $\mathrm{E} 4.4$ & - & - & $\mathrm{LC}$ \\
\hline 131 & Chenopodiaceae & Atriplex tatarica L. var. tatarica & $\mathrm{E} 4.4$ & - & - & $\mathrm{LC}$ \\
\hline 132 & Chenopodiaceae & $\begin{array}{l}\text { Camphorosma monspeliaca L. } \\
\text { subsp. monspeliaca }\end{array}$ & E2.5 & - & - & $\mathrm{LC}$ \\
\hline 133 & Chenopodiaceae & $\begin{array}{l}\text { Chenopodium album L. subsp. } \\
\text { album var. album }\end{array}$ & E2.5 & - & - & $\mathrm{LC}$ \\
\hline 134 & Chenopodiaceae & Chenopodium botrys L. & 11.3 & - & - & $\mathrm{LC}$ \\
\hline 135 & Chenopodiaceae & $\begin{array}{l}\text { Chenopodium foliosum } \\
\text { (Moench) Aschers' }\end{array}$ & $\mathrm{E} 4.4$ & - & - & $\mathrm{LC}$ \\
\hline 136 & Chenopodiaceae & $\begin{array}{l}\text { Cyathobasis fruticulosa (Bunge) } \\
\text { Aellen }\end{array}$ & E2.5 & Endemik & - & VU \\
\hline 137 & Chenopodiaceae & $\begin{array}{l}\text { Halimione verrucifera (Bieb.) } \\
\text { Aellen }\end{array}$ & $\mathrm{E} 4.4$ & - & - & $\mathrm{LC}$ \\
\hline 138 & Chenopodiaceae & $\begin{array}{l}\text { Halocnemum strobilaceum } \\
\text { (Pall.) Bieb. }\end{array}$ & $\mathrm{E} 2.5$ & - & - & $\mathrm{LC}$ \\
\hline 139 & Chenopodiaceae & Kochia prostrata (L.) Schrad. & E4.4 & - & - & $\mathrm{LC}$ \\
\hline 140 & Chenopodiaceae & $\begin{array}{l}\text { Krascheninnikovia ceroides (L.) } \\
\text { Güldenst. }\end{array}$ & $\mathrm{E} 2.5$ & - & - & $\mathrm{LC}$ \\
\hline 141 & Chenopodiaceae & $\begin{array}{l}\text { Noaea mucronata (Forssk.) } \\
\text { Aschers. \& Schvveinf subsp. } \\
\text { mucronata }\end{array}$ & $\mathrm{E} 4.4$ & - & - & $\mathrm{LC}$ \\
\hline 142 & Chenopodiaceae & Panderia pilosa Fisch. \& Mey. & E4.4 & - & - & $\mathrm{LC}$ \\
\hline 143 & Chenopodiaceae & $\begin{array}{l}\text { Petrosimonia brachiata (Pallas) } \\
\text { Bunge }\end{array}$ & $\mathrm{E} 2.5$ & - & 1 & $\mathrm{LC}$ \\
\hline 144 & Chenopodiaceae & $\begin{array}{l}\text { Salicornia europaea L. subsp. } \\
\text { prostrata Pall }\end{array}$ & E2.5 & - & - & $\mathrm{LC}$ \\
\hline 145 & Chenopodiaceae & Salsola inermis Forssk. & $\mathrm{E} 2.5$ & - & Ir.-Tur. ele. & $\mathrm{LC}$ \\
\hline 146 & Chenopodiaceae & Salsola laricina Pall. & $\mathrm{E} 2.5$ & - & - & $\mathrm{LC}$ \\
\hline 147 & Chenopodiaceae & Salsola stenoptera Wagenitz & $\mathrm{E} 2.5$ & Endemik & Ir.-Tur. ele. & $\mathrm{LC}$ \\
\hline 148 & Chenopodiaceae & Suaeda carnosissima Post & E2.5 & - & Ir.-Tur. ele. & $\mathrm{LC}$ \\
\hline 149 & Cistaceae & $\begin{array}{l}\text { Helianthemum nummularium } \\
\text { (L.) Miller subsp. nummitlariım }\end{array}$ & $\mathrm{E} 4.4$ & - & - & $\mathrm{LC}$ \\
\hline 150 & Cistaceae & $\begin{array}{l}\text { Helianthemum salicifolium (L.) } \\
\text { Miller }\end{array}$ & E4.4 & & - & $\mathrm{LC}$ \\
\hline 151 & Convolvulaceae & Convolvulus arvensis L. & $\mathrm{E} 1.2$ & - & - & $\mathrm{LC}$ \\
\hline 152 & Convolvulaceae & Convolvulus assyriacus Griseb. & $\mathrm{E} 4.4$ & Endemik & Ir.-Tur. ele. & $\mathrm{LC}$ \\
\hline 153 & Convolvulaceae & $\begin{array}{l}\text { Convolvulus galaticus Rostan } \\
\text { ex Choisy }\end{array}$ & $\mathrm{E} 4.4$ & Endemik & Ir.-Tur. ele. & $\mathrm{LC}$ \\
\hline
\end{tabular}




\begin{tabular}{|c|c|c|c|c|c|c|}
\hline 154 & Convolvulaceae & $\begin{array}{l}\text { Convolvulus holosericeus Bieb. } \\
\text { subsp. holosericeus }\end{array}$ & E4.4 & - & - & $\mathrm{LC}$ \\
\hline 155 & Convolvulaceae & Convolvulus lineatus L. & $\mathrm{E} 4.4$ & - & . & $\mathrm{LC}$ \\
\hline 156 & Crassulaceae & Sedum acre L. & H3.6 & - & - & $\mathrm{LC}$ \\
\hline No & Family & Species & $\begin{array}{l}\text { EUNIS Habitat } \\
\text { code }\end{array}$ & Endemism & $\begin{array}{l}\text { Phytogeographical } \\
\text { Region }\end{array}$ & $\begin{array}{l}\text { Endangerment } \\
\text { statue }\end{array}$ \\
\hline 157 & Crassulaceae & Sedum album $\mathrm{L}$. & H3.6 & - & - & LC \\
\hline 158 & Cuscutaceae & Cuscuta campestris Yuncker & 11.3 & - & - & $\mathrm{LC}$ \\
\hline 159 & Dipsacaceae & Dipsctcus laciniatus L. & E2.5 & - & - & LC \\
\hline 160 & Dipsacaceae & Scabiosa argentea $\mathrm{L}$, & E4.4 & - & - & LC \\
\hline 161 & Dipsacaceae & Scabiosa calocephala Boiss. & E4.4 & - & Ir.-Tur. ele. & $\mathrm{LC}$ \\
\hline 162 & Dipsacaceae & Scabiosa rotata Bieb. & E4.4 & - & Ir.-Tur, ele. & LC \\
\hline 163 & Euphorbiceae & $\begin{array}{l}\text { Euphorbia anacampseros Boiss. } \\
\text { var. anacampseros }\end{array}$ & $\mathrm{E} 4.4$ & Endemik & - & LC \\
\hline 164 & Euphorbiceae & $\begin{array}{l}\text { Euphorbia arvalis Boiss. \& } \\
\text { Heldr. }\end{array}$ & E4.4 & - & Ir.Tur.ele. & $\mathrm{LC}$ \\
\hline 165 & Euphorbiceae & $\begin{array}{l}\text { Euphorbia falcata } \text { L. s11bsp. } \\
\text { falcata var. falcata }\end{array}$ & $\mathrm{E} 4.4$ & - & - & LC \\
\hline 166 & Euphorbiceae & Euphorbia macroclada Boiss. & E4.4 & - & Ir.Turi & $\mathrm{LC}$ \\
\hline 167 & Euphorbiceae & $\begin{array}{l}\text { Euphorbia szovitsii Fisch. \& } \\
\text { Mey. var. szovitsii }\end{array}$ & $\mathrm{E} 4.4$ & - & Ir.Tur. & $\mathrm{LC}$ \\
\hline 168 & Fabaceae & $\begin{array}{l}\text { Alhagi pseudolhagi (Bieb.) } \\
\text { Desv. }\end{array}$ & El.2 & - & Ir.-Tur. ele. & $\mathrm{LC}$ \\
\hline 169 & Fabaceae & $\begin{array}{l}\text { Astragalus angustifolius Lam. } \\
\text { subsp. longidens Hub.-Mor. }\end{array}$ & $\mathrm{E} 4.4$ & - & - & $\mathrm{LC}$ \\
\hline 170 & Fabaceae & Astragalus condensatus Ledeb. & H3.6 & Endemik & Ir.-Tur. ele. & $\mathrm{LC}$ \\
\hline 171 & Fabaceae & $\begin{array}{l}\text { Astragalus karamasicas Boiss. } \\
\text { \& Bal. }\end{array}$ & $\mathrm{E} 4.4$ & Endemik & Ir.-Tur. ele. & $\mathrm{LC}$ \\
\hline 172 & Fabaceae & $\begin{array}{l}\text { Astragalus ktrshehiriculs } \\
\text { Chamberlain }\end{array}$ & E4.4 & Endemik & Ir.-Tur. ele. & VU \\
\hline 173 & Fabaceae & Astragalus lycius Boiss. & E1.2 & Endemik & Ir.-Tur. ele. & $\mathrm{LC}$ \\
\hline 174 & Fabaceae & Astragalus lydius Boiss. & $\mathrm{E} 4.4$ & Endemik & Ir.-Tur. ele. & $\mathrm{LC}$ \\
\hline 175 & Fabaceae & Astragalus tokatensis Fischer & H3.6 & Endemik & Ir.-Tur. ele. & NT \\
\hline 176 & Fabaceae & Cicer arietinum $\mathrm{L}$. & 11.3 & - & • & LC \\
\hline 177 & Fabaceae & Coronilla varia L. subsp. varla & El.2 & - & - & $\mathrm{LC}$ \\
\hline 178 & Fabaceae & $\begin{array}{l}\text { Ebenus laguroides Boiss. var. } \\
\text { laguroides }\end{array}$ & H3.6 & Endemik & Ir.-Tur. ele. & LC \\
\hline 179 & Fabaceae & $\begin{array}{l}\text { Lathyrus aphaca L. var. biflorus } \\
\text { Post. }\end{array}$ & E1.2 & - & - & $\mathrm{LC}$ \\
\hline 180 & Fabaceae & Lens culinaris Medik. & 11.3 & • & - & $\mathrm{LC}$ \\
\hline 181 & Fabaceae & $\begin{array}{l}\text { Lotus corniculalus L. var. } \\
\text { corniculatus }\end{array}$ & El.2 & - & - & $\mathrm{LC}$ \\
\hline 182 & Fabaceae & $\begin{array}{l}\text { Medicago sativa } \mathrm{L} \text {. subsp. } \\
\text { sativa }\end{array}$ & 11.3 & - & - & $\mathrm{LC}$ \\
\hline 183 & Fabaceae & Melilotus officinalis (L.) Desr. & El.2 & - & - & $\mathrm{LC}$ \\
\hline 184 & Fabaceae & $\begin{array}{l}\text { Onobrychis armena Boiss. \& } \\
\text { Huet. }\end{array}$ & E4.4 & Endemik & - & $\mathrm{LC}$ \\
\hline 185 & Fabaceae & $\begin{array}{l}\text { Onobrychis montana DC. } \\
\text { subsp. cadmea (Boiss.) } \\
\text { P.W.Ball. }\end{array}$ & 11.3 & - & - & $\mathrm{LC}$ \\
\hline 186 & Fabaceae & $\begin{array}{l}\text { Ononis spinosa L. subsp. } \\
\text { antiquorum (L.) Briq. }\end{array}$ & E2.5 & - & Akd. ele. & $\mathrm{LC}$ \\
\hline
\end{tabular}




\begin{tabular}{|c|c|c|c|c|c|c|}
\hline 187 & Fabaceae & Phaseolus vulgaris L. & 11.3 & - & - & $\mathrm{LC}$ \\
\hline 188 & Fabaceae & $\begin{array}{l}\text { Tetragonolobus maritimus (L.) } \\
\text { Roth }\end{array}$ & El.2 & - & - & $\mathrm{LC}$ \\
\hline 189 & Fabaceae & $\begin{array}{l}\text { Trifolium arvense } \mathrm{L} . \mathrm{var} . \\
\text { arvense }\end{array}$ & El.2 & - & - & $\mathrm{LC}$ \\
\hline No & Family & Species & $\begin{array}{l}\text { EUNIS Habitat } \\
\text { code }\end{array}$ & Endemism & $\begin{array}{l}\text { Phytogeographical } \\
\text { Region }\end{array}$ & $\begin{array}{l}\text { Endangerment } \\
\text { statue }\end{array}$ \\
\hline 190 & Fabaceae & Trifolium campestre Schreb. & El.2 & - & - & LC \\
\hline 191 & Fabaceae & $\begin{array}{l}\text { Trifolium physodes Stev. ex } \\
\text { Bieb. var. physodes }\end{array}$ & E1.2 & - & Akd. ele. & $\mathrm{LC}$ \\
\hline 192 & Fabaceae & $\begin{array}{l}\text { Trifolium repens L. var. } \\
\text { macrorrhizum (Boiss.) Boiss. }\end{array}$ & El.2 & - & - & $\mathrm{LC}$ \\
\hline 193 & Fabaceae & $\begin{array}{l}\text { Vicia cracca } \text { L. subsp. } \\
\text { stenophylla } \text { Vel. }\end{array}$ & E1.2 & - & - & $\mathrm{LC}$ \\
\hline 194 & Fabaceae & Vicia erillea $($ L.) Willd. & E1.2 & & - & $\mathrm{LC}$ \\
\hline 195 & Frankeniaceae & Frankenia hirsuta $\mathrm{L}$. & $\mathrm{E} 2.5$ & - & - & $\mathrm{LC}$ \\
\hline 196 & Gentianaceae & $\begin{array}{l}\text { Centaurium erythraea Rafn } \\
\text { subsp. turcicum (Velen.) } \\
\text { Melderis }\end{array}$ & $\mathrm{E} 4.4$ & - & - & $\mathrm{LC}$ \\
\hline 197 & Geraniaceae & $\begin{array}{l}\text { Erodium cicutarium (L.) } \\
\text { L'Herit. subsp. dcutarium }\end{array}$ & E4.4 & - & - & $\mathrm{LC}$ \\
\hline 198 & Geraniaceae & $\begin{array}{l}\text { Geranium collinum Steph. ex } \\
\text { Willd, }\end{array}$ & E2.5 & & - & $\mathrm{LC}$ \\
\hline 199 & Globulariaceae & Globularia orientalis L. & H3.6 & & Ir.-Tur. ele. & LC \\
\hline 200 & Globulariaceae & $\begin{array}{l}\text { Globularia trichosantha Fisch. } \\
\text { \& Mey. }\end{array}$ & H3.6 & - & Ir.-Tur. ele. & $\mathrm{LC}$ \\
\hline 201 & Hypericaceae & Hypericum perforatum $\mathrm{L}$. & 11.3 & - & - & $\mathrm{LC}$ \\
\hline 202 & Illecebraceae & $\begin{array}{l}\text { Paronychia kurdica Boiss. } \\
\text { subsp. kurdica var. kurdica }\end{array}$ & $\mathrm{E} 4.4$ & - & - & $\mathrm{LC}$ \\
\hline 203 & Juglandaceae & Juglans regia $\mathrm{L}$. & $\mathrm{E} 4.4$ & - & - & $\mathrm{LC}$ \\
\hline 204 & Lamiaceae & $\begin{array}{l}\text { Ajuga chamaepitya }(\mathrm{L} .) \\
\text { Schreber subsp. chia }(\text { Schreber }) \\
\text { Arcangeli }\end{array}$ & $\mathrm{E} 4.4$ & - & - & $\mathrm{LC}$ \\
\hline 205 & Lamiaceae & Lamium amplexicule $\mathrm{L}$. & $\mathrm{E} 4.4$ & - & Avr. -Sib. ele. & $\mathrm{LC}$ \\
\hline 206 & Lamiaceae & $\begin{array}{l}\text { Marrubium astracanicum Jacq. } \\
\text { subsp. astracanicum }\end{array}$ & E4.4 & - & - & $\mathrm{LC}$ \\
\hline 207 & Lamiaceae & $\begin{array}{l}\text { Marrubium parviflorum Fisch. } \\
\text { \& Mey. subsp. oliodon (Boiss.) } \\
\text { Seybold }\end{array}$ & E4.4 & Endemik & - & LÇ \\
\hline 208 & Lamiaceae & $\begin{array}{l}\text { Mentha longifolia (L.) Hudson } \\
\text { subsp. typhoides (Briq) Harley }\end{array}$ & $\mathrm{E} 2.5$ & - & - & $\mathrm{LC}$ \\
\hline 209 & Lamiaceae & $\begin{array}{l}\text { Nepeta stricta (Banks \& Sol) } \\
\text { Hedge \& Lamond var. } \\
\text { curvidens (Boiss. \& Bal.) Hedge } \\
\text { \& Lamond }\end{array}$ & 11.3 & - & Ir.-Tur. ele. & $\mathrm{LC}$ \\
\hline 210 & Lamiaceae & Phlomis armeniaca Willd. & E4.4 & Endemik & Ir.-Tur. ele. & $\mathrm{LC}$ \\
\hline 211 & Lamiaceae & $\begin{array}{l}\text { Phlomis pungens Willd. var. } \\
\text { hirta Velen }\end{array}$ & $\mathrm{E} 4.4$ & & - & $\mathrm{LC}$ \\
\hline 212 & Lamiaceae & Salvia verbenaca $\mathrm{L}$. & E4.4 & & - & $\mathrm{LC}$ \\
\hline 213 & Lamiaceae & $\begin{array}{l}\text { Sideritis montana L. subsp. } \\
\text { montana }\end{array}$ & $\mathrm{E} 4.4$ & - & Akd. ele. & $\mathrm{LC}$ \\
\hline 214 & Lamiaceae & Stachys byzantina C. Koclı & $\mathrm{E} 4.4$ & - & Avr. -Sib. ele. & $\mathrm{LC}$ \\
\hline 215 & Lamiaceae & $\begin{array}{l}\text { Stachys amma (L.) L. subsp. } \\
\text { annua var. anпиa }\end{array}$ & E4.4 & - & - & $\mathrm{LC}$ \\
\hline
\end{tabular}




\begin{tabular}{|c|c|c|c|c|c|c|}
\hline 216 & Lamiaceae & $\begin{array}{l}\text { Stachys lavandulifolia Vahl var. } \\
\text { alavandulifolia }\end{array}$ & H3.6 & - & Ir.-Tur. ele. & $\mathrm{LC}$ \\
\hline 217 & Lamiaceae & $\begin{array}{l}\text { Teucrium chamaediys L. subsp. } \\
\text { chamaedrys }\end{array}$ & E4.4 & - & Avr. -Sib. ele. & $\mathrm{LC}$ \\
\hline 218 & Lamiaceae & Teucrium polium $L$ & $\mathrm{E} 4.4$ & & ' & $\mathrm{LC}$ \\
\hline No & Family & Species & $\begin{array}{l}\text { EUNIS Habitat } \\
\text { code }\end{array}$ & Endemism & $\begin{array}{l}\text { Phytogeographical } \\
\text { Region }\end{array}$ & $\begin{array}{l}\text { Endangerment } \\
\text { statue }\end{array}$ \\
\hline 219 & Lamiaceae & $\begin{array}{l}\text { Teucrium scordium L. subsp. } \\
\text { scordioides (Schreber) Maire }\end{array}$ & E4.4 & - & - & $\mathrm{LC}$ \\
\hline 220 & Lamiaceae & $\begin{array}{l}\text { Thymus sipyleus Boiss. subsp. } \\
\text { rosulans (Borbas) Jalas }\end{array}$ & $\mathrm{E} 4.4$ & - & - & $\mathrm{LC}$ \\
\hline 221 & Lamiaceae & $\begin{array}{l}\text { Wiedemannia orientalis Fisch. } \\
\& \text { Mey. }\end{array}$ & 11.3 & - & Ir.-Tur. ele. & $\mathrm{LC}$ \\
\hline 222 & Lamiaceae & $\begin{array}{l}\text { Ziziphora taurica Bieb. subsp. } \\
\text { taurica }\end{array}$ & H3.6 & & Ir.-Tur. ele. & $\mathrm{LC}$ \\
\hline 223 & Lamiaceae & Ziziphora tenuior L. & E4.4 & - & Ir.-Tur. ele. & $\mathrm{LC}$ \\
\hline 224 & Linaceae & $\begin{array}{l}\text { Linum austriacum L. subsp. } \\
\text { austriacum }\end{array}$ & $\mathrm{E} 4.4$ & - & - & $\mathrm{LC}$ \\
\hline 225 & Lythraceae & Lythrum salicaria $\mathrm{L}$. & $\mathrm{E} 2.5$ & - & Avr. -Sib. ele. & $\mathrm{LC}$ \\
\hline 226 & Malvaceae & $\begin{array}{l}\text { Alcea aplerocarpa (Fenzl) } \\
\text { Boiss. }\end{array}$ & $\mathrm{E} 4.4$ & Endemik & Ir.-Tur. ele. & $\mathrm{LC}$ \\
\hline 227 & Malvaceae & Malva neglecta Wallr. & $\mathrm{E} 4.4$ & - & - & $\mathrm{LC}$ \\
\hline 228 & Moraceae & Morus alba L. & E4.4 & & - & $\mathrm{LC}$ \\
\hline 229 & Moraceae & Morus nigra L. & $\mathrm{E} 4.4$ & - & - & $\mathrm{LC}$ \\
\hline 230 & Oleaceae & Fraxinus angustifolia Vahi & $\mathrm{E} 4.4$ & - & - & $\mathrm{LC}$ \\
\hline 231 & Onagraceae & Epilobium montanum L. & E2.5 & - & Avr. -Sib. ele. & $\mathrm{LC}$ \\
\hline 232 & Orobanchaceae & $\begin{array}{l}\text { Orobanche anatolica Boiss. \& } \\
\text { Reuter }\end{array}$ & $\mathrm{E} 4.4$ & - & - & $\mathrm{LC}$ \\
\hline 232 & Papaveraceae & Fumaria asepala Boiss. & $\mathrm{E} 4.4$ & - & - & $\mathrm{LC}$ \\
\hline 233 & Papaveraceae & Glacium leiocarpum Boiss. & $\mathrm{E} 4.4$ & - & - & $\mathrm{LC}$ \\
\hline 234 & Papaveraceae & $\begin{array}{l}\text { Papaver commutatum Fisch. \& } \\
\text { Mey. }\end{array}$ & $\mathrm{E} 4.4$ & - & - & $\mathrm{LC}$ \\
\hline 235 & Papaveraceae & Papaver rhoeas L. & $\mathrm{E} 4.4$ & - & - & $\mathrm{LC}$ \\
\hline 236 & Plantaginaceae & Plantago atrata Hoppe & $\mathrm{E} 2.5$ & - & - & $\mathrm{LC}$ \\
\hline 237 & Plantaginaceae & Plantago holosteum Scop. & $\mathrm{E} 2.5$ & - & - & $\mathrm{LC}$ \\
\hline 238 & Plantaginaceae & Plantago lanceolata L. & El.2 & - & - & $\mathrm{LC}$ \\
\hline 239 & Plantaginaceae & $\begin{array}{l}\text { Plantago major L. subsp. } \\
\text { intermedia (Gilib.) Lange }\end{array}$ & E2.5 & - & - & $\mathrm{LC}$ \\
\hline 240 & Plumbaginaceae & $\begin{array}{l}\text { Acantholimon acerosum } \\
\text { (Willd.) Boiss. var. acerosum }\end{array}$ & $\mathrm{E} 4.4$ & - & Ir.Tur. ele. & $\mathrm{LC}$ \\
\hline 241 & Polygalaceae & $\begin{array}{l}\text { Polygala pruinosa Boiss. subsp. } \\
\text { pruinosa }\end{array}$ & E2.5 & - & - & $\mathrm{LC}$ \\
\hline 242 & Polygonaceae & Polygonum arenastrum Bar. & E4.4 & - & - & $\mathrm{LC}$ \\
\hline 243 & Polygonaceae & Polygonum bellardii Ali. & $\mathrm{E} 4.4$ & - & - & $\mathrm{LC}$ \\
\hline 244 & Polygonaceae & $\begin{array}{l}\text { Polygonum polycnemoides } \\
\text { Jaub. \& Spach }\end{array}$ & $\mathrm{E} 4.4$ & - & Ir.-Tur. ele. & $\mathrm{LC}$ \\
\hline 245 & Polygonaceae & Rumex acetocella $\mathrm{L}$. & $\mathrm{E} 4.4$ & - & - & $\mathrm{LC}$ \\
\hline 246 & Polygonaceae & Rumex crispus L. & E2.5 & - & - & $\mathrm{LC}$ \\
\hline 247 & Primulaceae & $\begin{array}{l}\text { Anagallis arvensis L. var. } \\
\text { arvensis }\end{array}$ & $\mathrm{E} 4.4$ & - & - & $\mathrm{LC}$ \\
\hline 248 & Primulaceae & Androsace maxima L. & E4.4 & - & - & $\mathrm{LC}$ \\
\hline
\end{tabular}




\begin{tabular}{|c|c|c|c|c|c|c|}
\hline 249 & Primulaceae & Androsace villosa $\mathrm{L}$. & $\mathrm{E} 4.4$ & - & - & $\mathrm{LC}$ \\
\hline 250 & Primulaceae & Glaux maritima L. & E2.5 & - & - & $\mathrm{LC}$ \\
\hline 251 & Ranunculaceae & $\begin{array}{l}\text { Adonis aestivalis L. subsp. } \\
\text { aestivalis }\end{array}$ & E4.4. & - & - & $\mathrm{LC}$ \\
\hline No & Family & Species & $\begin{array}{l}\text { EUNIS Habitat } \\
\text { code }\end{array}$ & Endemism & $\begin{array}{l}\text { Phytogeographical } \\
\text { Region }\end{array}$ & $\begin{array}{l}\text { Endangerment } \\
\text { statue }\end{array}$ \\
\hline 252 & Ranunculaceae & $\begin{array}{l}\text { Ceratocephalus falcatus (L.) } \\
\text { Pers. }\end{array}$ & E4.4 & - & - & $\mathrm{LC}$ \\
\hline 253 & Ranunculaceae & $\begin{array}{l}\text { Consolida glandulosa (Boiss. \& } \\
\text { Huet) Bornm. }\end{array}$ & $\mathrm{E} 4.4$ & Endemik & Ir.-Tur. ele. & $\mathrm{LC}$ \\
\hline 254 & Ranunculaceae & $\begin{array}{l}\text { Consolida hellespontica } \\
\text { (Boiss.) Chater }\end{array}$ & E4.4 & - & Ir.-Tur. ele. & $\mathrm{LC}$ \\
\hline 255 & Ranunculaceae & $\begin{array}{l}\text { Consolida orientalis (Gay) } \\
\text { Schröd }\end{array}$ & E4.4 & - & Ir.-Tur. ele. & LC \\
\hline 256 & Ranunculaceae & $\begin{array}{l}\text { Nigella arvensis L. var. glauca } \\
\text { Boiss. }\end{array}$ & $\mathrm{E} 4.4$ & - & - & \\
\hline 257 & Ranunculaceae & Nigella segetalis Bieb. & $\mathrm{E} 4.4$ & - & - & $\mathrm{LC}$ \\
\hline 258 & Ranunculaceae & Ranunculus arvensis L. & E2.5 & - & - & LC \\
\hline 259 & Ranunculaceae & $\begin{array}{l}\text { Ranunculus } \\
\text { constantinopolitanus (DC.) } \\
\text { d'Urv, }\end{array}$ & $\mathrm{E} 2.5$ & - & - & $\mathrm{LC}$ \\
\hline 260 & Ranunculaceae & Ranunculus scleratus L. & $\mathrm{E} 2.5$ & & - & LC \\
\hline 261 & Ranunculaceae & Thalictrum minus L. var. minus & $\mathrm{E} 2.5$ & - & - & $\mathrm{LC}$ \\
\hline 262 & Resedaceae & Reseda lutea L. var, lutea & $\mathrm{E} 4.4$ & - & - & LC \\
\hline 263 & Rosaceae & Amygdalus communis $\mathrm{L}$. & E4.4 & . & - & $\mathrm{LC}$ \\
\hline 264 & Rosaceae & Cerasus duhamel (L.) Moench & E4.4 & - & - & $\mathrm{LC}$ \\
\hline 265 & Rosaceae & Cerasıs vulgaris Miller & E4.4 & - & - & $\mathrm{LC}$ \\
\hline 266 & Rosaceae & Cydonia oblonga Miller & $\mathrm{E} 4.4$ & . & - & $\mathrm{LC}$ \\
\hline 267 & Rosaceae & Malus sylvestris Miller & E4.4 & - & - & LC \\
\hline 268 & Rosaceae & Potentilla recta $\mathrm{L}$. & E1.2 & - & - & $\mathrm{LC}$ \\
\hline 269 & Rosaceae & Potentilla reptans $\mathrm{L}$. & E1.2 & - & - & $\mathrm{LC}$ \\
\hline 270 & Rosaceae & Prunus xdomestica L. & E4.4 & - & - & $\mathrm{LC}$ \\
\hline 271 & Rosaceae & Pyrus communis L. & E4.4 & - & - & $\mathrm{LC}$ \\
\hline 272 & Rosaceae & Rosa canina $\mathrm{L}$. & $\mathrm{E} 4.4$ & - & - & $\mathrm{LC}$ \\
\hline 273 & Rosaceae & $\begin{array}{l}\text { Sanguisorba minör Scop. subsp. } \\
\text { muricata (Spach) Briq. }\end{array}$ & E1.2 & - & - & $\mathrm{LC}$ \\
\hline 274 & Rubiaceae & Crucianella distachia Boiss. & E4.4 & Endemik & Avr. -Sib. ele. & $\mathrm{LC}$ \\
\hline 275 & Rubiaceae & $\begin{array}{l}\text { Cruciata taurica (Pallas ex } \\
\text { Willd.) Ehrend. }\end{array}$ & H3.6 & - & Ir.-Tur. ele. & LC. \\
\hline 276 & Rubiaceae & Galium aparine $\mathrm{L}$. & E1.2 & - & - & $\mathrm{LC}$ \\
\hline 277 & Rubiaceae & $\begin{array}{l}\text { Galium incanum Sm. subsp. } \\
\text { elatius (Boiss.) Ehrend. }\end{array}$ & H3.6 & - & Ir.-Tur, ele. & $\mathrm{LC}$ \\
\hline 278 & Rubiaceae & Galium verum L. subsp. verum & E1.2 & - & Avr. -Sib. ele. & $\mathrm{LC}$ \\
\hline 279 & Salicaceae & Populus alba $\mathrm{L}$. & $\mathrm{E} 4.4$ & - & Avr. -Sib. ele. & $\mathrm{LC}$ \\
\hline 280 & Salicaceae & Populus nigra L. & $\mathrm{E} 4.4$ & - & - & $\mathrm{LC}$ \\
\hline 281 & Salicaceae & Salix alba $\mathrm{L}$. & E4.4 & - & Avr. -Sib. ele. & $\mathrm{LC}$ \\
\hline 282 & Scrophulariaceae & $\begin{array}{l}\text { Bungea trifida (Vahl) } \\
\text { C.A.meyer }\end{array}$ & $\mathrm{E} 4.4$ & - & Ir.-Tur. ele. & $\mathrm{LC}$ \\
\hline 283 & Scrophulariaceae & Linaria corifolia Desf. & H3.6 & Endemik & Ir.-Tur. ele. & $\mathrm{LC}$ \\
\hline
\end{tabular}




\begin{tabular}{|c|c|c|c|c|c|c|}
\hline 284 & Scrophulariaceae & $\begin{array}{l}\text { Linaria genistifolia (L.) Miller } \\
\text { subsp. confertiflora (Boiss.) } \\
\text { Davis }\end{array}$ & 11.3 & Endemik & Ir.-Tur. ele. & $\mathrm{LC}$ \\
\hline 285 & Scrophulariaceae & $\begin{array}{l}\text { Linaria kurdica Boiss. \& Hohen } \\
\text { subsp. aucheri (Boiss.) Davis }\end{array}$ & 11.3 & - & Ir.-Tur. ele. & $\mathrm{LC}$ \\
\hline No & Family & Species & $\begin{array}{l}\text { EUNIS Habitat } \\
\text { code }\end{array}$ & Endemism & $\begin{array}{l}\text { Phytogeographical } \\
\text { Region }\end{array}$ & $\begin{array}{l}\text { Endangerment } \\
\text { statue }\end{array}$ \\
\hline 286 & Scrophulariaceae & Odontites aucheri Boiss. & E2.5 & . & Ir.-Tur. ele. & $\mathrm{LC}$ \\
\hline 287 & Scrophulariaceae & Verbascum armenum L. & $\mathrm{E} 4.4$ & - & - & $\mathrm{LC}$ \\
\hline 288 & Scrophulariaceae & $\begin{array}{l}\text { Verbascum cherianthiflium } \\
\text { Boiss. var. cherianthifolium }\end{array}$ & $\mathrm{E} 2.5$ & - & - & $\mathrm{LC}$ \\
\hline 289 & Scrophulariaceae & $\begin{array}{l}\text { Verbascum vulcanicum Boiss. } \\
\text { \& Heldr. var. vulcanicum }\end{array}$ & $\mathrm{E} 2.5$ & Endemik & Ir.-Tur. ele. & $\mathrm{LC}$ \\
\hline 290 & Scrophulariaceae & Veronica anagallis-aquatica L. & E2.5 & - & & $\mathrm{LC}$ \\
\hline 291 & Scrophulariaceae & Veronica ersica Poiret & $\mathrm{E} 2.5$ & - & - & $\mathrm{LC}$ \\
\hline 292 & Scrophulariaceae & $\begin{array}{l}\text { Veronica grisebachii } \\
\text { S.M.Walters }\end{array}$ & $\mathrm{E} 1.2$ & - & Akd.ele. & $\mathrm{LC}$ \\
\hline 293 & Scrophulariaceae & Veronica multifida L. & El.2 & - & - & $\mathrm{LC}$ \\
\hline 294 & Scrophulariaceae & $\begin{array}{l}\text { Veronica orientalis Miller } \\
\text { subsp. nimrodi (Richter ex } \\
\text { Stapf) M.A.Fischer }\end{array}$ & El.2 & Endemik & - & $\mathrm{LC}$ \\
\hline 295 & Scrophulariaceae & Veronica verna $\mathrm{L}$. & E2.5 & - & Avr. -Sib. ele. & $\mathrm{LC}$ \\
\hline 296 & Solanaceae & Hyoscyamus niger L. & $\mathrm{E} 1.2$ & - & - & $\mathrm{LC}$ \\
\hline 297 & Solanaceae & Hyoscyamus reticulatus L. & $\mathrm{El} .2$ & - & Ir.-Tur. ele. & $\mathrm{LC}$ \\
\hline 298 & Solanaceae & $\begin{array}{l}\text { Lycium anatolicum A. Baytop } \\
\text { \& R. Mili }\end{array}$ & $\mathrm{E} 4.4$ & Endemik & Ir.-Tur. ele. & $\mathrm{LC}$ \\
\hline 299 & Solanaceae & Lycium depressum Stocks & E4.4 & - & Ir.Tur. & $\mathrm{LC}$ \\
\hline 300 & Tamaricaceae & $\begin{array}{l}\text { Reaumuria aliernifolia (Lab.) } \\
\text { Britten }\end{array}$ & $\mathrm{E} 4.4$ & - & Ir.-Tur. ele. & $\mathrm{LC}$ \\
\hline 301 & Thymalaeaceae & $\begin{array}{l}\text { Thymelaea passerina (L.) } \\
\text { Cosson \& Germ. }\end{array}$ & $\mathrm{E} 4.4$ & - & - & $\mathrm{LC}$ \\
\hline 302 & Ulmaceae & $\begin{array}{l}\text { Ulmus minör Miller subsp. } \\
\text { minor }\end{array}$ & E4.4 & - & - & $\mathrm{LC}$ \\
\hline 303 & Valerianaceae & Valerianella coronata (L.) DC. & $\mathrm{E} 4.4$ & - & - & $\mathrm{LC}$ \\
\hline 304 & Vitaceae & Vitis vinifra $\mathrm{L}$. & E4.4 & & & $\mathrm{LC}$ \\
\hline 305 & Zygophyllaceae & Peganum harmala L. & $\mathrm{E} 4.4$ & - & & $\mathrm{LC}$ \\
\hline 306 & Zygophyllaceae & Tribuhts terrestris L. & $\mathrm{E} 1.2$ & - & & $\mathrm{LC}$ \\
\hline \multirow[t]{2}{*}{307} & Zygophyllaceae & Zygophyllum fabago L. & $\mathrm{E} 4.4$ & - & Ir.-Tur. ele. & $\mathrm{LC}$ \\
\hline & $\begin{array}{l}\text { MONOCOTYLEDO } \\
\text { NES }\end{array}$ & & & & & \\
\hline 308 & Alismataceae & Alisma pIantago-aqualica L. & D6.1 & & Avr. -Sib. ele. & $\mathrm{LC}$ \\
\hline 309 & Butomaceae & Butomus umbellatus L. & D6.1 & - & Avr. -Sib. ele. & $\mathrm{LC}$ \\
\hline 310 & Cyperaceae & $\begin{array}{l}\text { Bolboschoenus maritimus (L.) } \\
\text { Palla var. maritimus }\end{array}$ & D6.1 & - & - & $\mathrm{LC}$ \\
\hline 311 & Cyperaceae & Carex acutiformis Ehrh. & $\mathrm{E} 1.2$ & & Avr. -Sib. ele. & $\mathrm{LC}$ \\
\hline 312 & Cyperaceae & $\begin{array}{l}\text { Carex cilicica Boiss. subsp. } \\
\text { cilicica }\end{array}$ & El.2 & - & Ir.-Tur. ele. & $\mathrm{LC}$ \\
\hline 313 & Cyperaceae & Carex dis/ans L. & E2.5 & - & - & $\mathrm{LC}$ \\
\hline 314 & Cyperaceae & Carex divisa Hudson & $\mathrm{E} 1.2$ & - & Avr. -Sib. ele. & $\mathrm{LC}$ \\
\hline 315 & Cyperaceae & $\begin{array}{l}\text { Carex nigra (L.) Reichd subsp. } \\
\text { dacica (Heuffel) Soo }\end{array}$ & $\mathrm{E} 1.2$ & - & Öksin ele. & $\mathrm{LC}$ \\
\hline 316 & Cyperaceae & Carex otrubae Podp. & El.2 & & Avr. -Sib. ele. & $\mathrm{LC}$ \\
\hline
\end{tabular}

49 | $\mathrm{P}$ a g e 


\begin{tabular}{|c|c|c|c|c|c|c|}
\hline 317 & Cyperaceae & Carex riparia Curtis & El.2 & - & Avr. -Sib. ele. & $\mathrm{LC}$ \\
\hline 318 & Cyperaceae & Eleocharis mitrocarpa Steudel & El.2 & - & - & $\mathrm{LC}$ \\
\hline 319 & Cyperaceae & $\begin{array}{l}\text { Eleocharis uniglumis (Link) } \\
\text { Schultes }\end{array}$ & D6.1 & - & - & $\mathrm{LC}$ \\
\hline No & Family & Species & $\begin{array}{l}\text { EUNIS Habitat } \\
\text { code }\end{array}$ & Endemism & $\begin{array}{l}\text { Phytogeographical } \\
\text { Region }\end{array}$ & $\begin{array}{l}\text { Endangerment } \\
\text { statue }\end{array}$ \\
\hline 320 & Cyperaceae & $\begin{array}{l}\text { Schoenoplectus lacustris (L.) } \\
\text { Palla }\end{array}$ & D6.1 & - & - & $\mathrm{LC}$ \\
\hline 321 & Cyperaceae & $\begin{array}{l}\text { Schoenoplectus supinus (L.) } \\
\text { Palla }\end{array}$ & D6.1 & - & - & $\mathrm{LC}$ \\
\hline 322 & Cyperaceae & $\begin{array}{l}\text { Scirpoides holoschoenus (L.) } \\
\text { Sojak }\end{array}$ & E2.5 & - & - & $\mathrm{LC}$ \\
\hline 323 & Juncaceae & $\begin{array}{l}\text { Juncus gerardi Loisel subsp. } \\
\text { gerardi }\end{array}$ & El.2 & - & - & $\mathrm{LC}$ \\
\hline 324 & Juncaceae & $\begin{array}{l}\text { Jucus heldreichianus Marsson } \\
\text { ex Parl. subsp. orientalis Snog }\end{array}$ & E1.2 & - & Ir.-Tur. ele. & $\mathrm{LC}$ \\
\hline 325 & Juncaginaceae & Triglochin palustris L. & El.2 & - & - & $\mathrm{LC}$ \\
\hline 326 & Iridaceae & Iris orientalis Miller & D6.1 & - & Akd. ele. & $\mathrm{LC}$ \\
\hline 327 & Liliaceae & Allium atroviolaceum Boiss. & 11.3 & - & - & $\mathrm{LC}$ \\
\hline 328 & Liliaceae & Allium cappadocicum Boiss. & $\mathrm{E} 4.4$ & Endemik & Ir.-Tur. ele. & $\mathrm{LC}$ \\
\hline 329 & Liliaceae & Allium cepa $\mathrm{L}$. & $\mathrm{E} 4.4$ & - & - & $\mathrm{LC}$ \\
\hline 330 & Liliaceae & Allium pseudoflavum Vved. & E4.4 & - & Ir.-Tur. ele. & $\mathrm{LC}$ \\
\hline 331 & Liliaceae & $\begin{array}{l}\text { Allium scorodoprasum L. subsp. } \\
\text { rotundum (L.) Stearn }\end{array}$ & H3.6 & - & Akd. ele. & $\mathrm{LC}$ \\
\hline 332 & Liliaceae & Allium vineale $\mathrm{L}$ & $\mathrm{E} 4.4$ & - & - & LC \\
\hline 333 & Liliaceae & Colchicum boissieri Orph. & $\mathrm{E} 2.5$ & - & Akd. ele. & $\mathrm{LC}$ \\
\hline 334 & Liliaceae & $\begin{array}{l}\text { Colchicum szovitsii Fisch. \& } \\
\text { Mey. }\end{array}$ & $\mathrm{E} 2.5$ & - & Ir.-Tur. ele. & $\mathrm{LC}$ \\
\hline 335 & Liliaceae & $\begin{array}{l}\text { Gagea villosa (Griseb.) Duby } \\
\text { var. villosa }\end{array}$ & 11.3 & - & Akd. ele. & $\mathrm{LC}$ \\
\hline 336 & Liliaceae & $\begin{array}{l}\text { Muscari caucasicum (Griseb.) } \\
\text { Baker }\end{array}$ & El.2 & - & Ir.Tur. & $\mathrm{LC}$ \\
\hline 337 & Orchidaceae & Orchis palustris Jacq. & El.2 & - & - & $\mathrm{LC}$ \\
\hline 338 & Poaceae & Aegilops cylindrica Host & 11.3 & - & Ir.-Tur. ele. & $\mathrm{LC}$ \\
\hline 339 & Poaceae & $\begin{array}{l}\text { Aegilops umbellulata } \\
\text { Zhukovslcy }\end{array}$ & $\mathrm{E} 4.4$ & - & Ir.-Tur. ele. & $\mathrm{LC}$ \\
\hline 340 & Poaceae & $\begin{array}{l}\text { Aeluropus littoralis (Gouan) } \\
\text { Parl }\end{array}$ & E1.2 & - & - & $\mathrm{LC}$ \\
\hline 341 & Poaceae & $\begin{array}{l}\text { Agropyron cristatum }(\mathrm{L} .) \\
\text { Gaertner subsp. pectinatum } \\
\text { (Bieb.) Tzvelev var. pectinatum }\end{array}$ & E1.2 & - & - & $\mathrm{LC}$ \\
\hline 342 & Poaceae & Alopecuris arundinaceus Poiret & E4.4 & - & Avr. -Sib. ele. & $\mathrm{LC}$ \\
\hline 343 & Poaceae & Apera intermedia Harkel & H3.6 & - & Ir.-Tur.. ele. & $\mathrm{LC}$ \\
\hline 344 & Poaceae & Bromus cappadocicus Boiss. & H3.6 & - & Ir.-Tur. ele. & $\mathrm{LC}$ \\
\hline 345 & Poaceae & $\begin{array}{l}\text { Broimıs japonicus Thunb. } \\
\text { Subsp. japonicus }\end{array}$ & $\mathrm{E} 4.4$ & - & - & $\mathrm{LC}$ \\
\hline 346 & Poaceae & Bromus squarrosus L. & E1.2 & - & - & $\mathrm{LC}$ \\
\hline 347 & Poaceae & Bromus sterilis L. & E4.4 & - & - & $\mathrm{LC}$ \\
\hline 348 & Poaceae & Bromus tectorum $\mathrm{L}$. & E4.4 & - & - & $\mathrm{LC}$ \\
\hline 349 & Poaceae & Bromus tomentellus Boiss. & $\mathrm{E} 4.4$ & - & Ir.-Tur. ele. & $\mathrm{LC}$ \\
\hline 350 & Poaceae & $\begin{array}{l}\text { Cynodon dactylon (L.) Pers. } \\
\text { var. villosus } \text { Regel }\end{array}$ & El.2 & - & - & $\mathrm{LC}$ \\
\hline
\end{tabular}




\begin{tabular}{|c|c|c|c|c|c|c|}
\hline 351 & Poaceae & $\begin{array}{l}\text { Dactylis glomerata L. subsp. } \\
\text { hispanica (Roth) Nyman }\end{array}$ & $\mathrm{E} 1.2$ & - & & $\mathrm{LC}$ \\
\hline 352 & Poaceae & Echinaria capitata (L.) Desf. & $\mathrm{E} 4.4$ & - & - & $\mathrm{LC}$ \\
\hline No & Family & Species & $\begin{array}{l}\text { EUNIS Habitat } \\
\text { code }\end{array}$ & Endemism & $\begin{array}{l}\text { Phytogeographical } \\
\text { Region }\end{array}$ & $\begin{array}{l}\text { Endangerment } \\
\text { statue }\end{array}$ \\
\hline 353 & Poaceae & $\begin{array}{l}\text { EIymus elongatus (Host) } \\
\text { Runemark s11bsp. ponticus } \\
\text { (Podp.) Melderis }\end{array}$ & El.2 & - & - & $\mathrm{LC}$ \\
\hline 354 & Poaceae & $\begin{array}{l}\text { EIymus lazicus (Boiss.) } \\
\text { Melderis subsp. divaricatus } \\
\text { (Boiss. \& Bal.) Melderis }\end{array}$ & E4.4 & Endemik & Ir.-Tur. ele. & $\mathrm{LC}$ \\
\hline 355 & Poaceae & $\begin{array}{l}\text { Eremopoa songarica (Schrenk) } \\
\text { Roshev. }\end{array}$ & El.2 & & Ir.-Tur. ele. & $\mathrm{LC}$ \\
\hline 356 & Poaceae & $\begin{array}{l}\text { Eremopyrum triticeum } \\
\text { (Gaertner) Nevski }\end{array}$ & $\mathrm{E} 4.4$ & - & - & $\mathrm{LC}$ \\
\hline 357 & Poaceae & $\begin{array}{l}\text { Festuca arundinacea } \text { Schreber } \\
\text { subsp. arundinacea }\end{array}$ & E1.2 & - & - & $\mathrm{LC}$ \\
\hline 358 & Poaceae & $\begin{array}{l}\text { Festuca callieri (Hackel ex St.- } \\
\text { Yves) F. Markgraf subsp. } \\
\text { callieri }\end{array}$ & E4.4 & - & - & $\mathrm{LC}$ \\
\hline 359 & Poaceae & $\begin{array}{l}\text { Festuca drymeja Mertens \& } \\
\text { Koch }\end{array}$ & $\mathrm{E} 1.2$ & - & Avr. -Sib. ele. & $\mathrm{LC}$ \\
\hline 360 & Poaceae & $\begin{array}{l}\text { Festuca valesiaca } \text { Schleischer } \\
\text { ex Gaudin }\end{array}$ & E4.4 & - & - & $\mathrm{LC}$ \\
\hline 361 & Poaceae & Hordeum bulbosum L. & 11.3 & - & - & $\mathrm{LC}$ \\
\hline 362 & Poaceae & $\begin{array}{l}\text { Hordeum murinum L. subsp. } \\
\text { glaucum (Steudel) Tzvelev }\end{array}$ & 11.3 & - & - & $\mathrm{LC}$ \\
\hline 363 & Poaceae & Koeleria cristata (L.) pers. & E4.4 & - & & $\mathrm{LC}$ \\
\hline 364 & Poaceae & Lolium perenne L. & E4.4 & - & Avr. -Sib. ele. & $\mathrm{LC}$ \\
\hline 365 & Poaceae & $\begin{array}{l}\text { Phleum exaratum Hochst. \& } \\
\text { Griseb subsp. exaratum }\end{array}$ & El.2 & - & - & $\mathrm{LC}$ \\
\hline 366 & Poaceae & $\begin{array}{l}\text { Phragmites australis (Cav.) } \\
\text { Trin. ex Steudel }\end{array}$ & D6.1 & - & Avr. -Sib. ele. & $\mathrm{LC}$ \\
\hline 367 & Poaceae & Poa bulbosa L. & E4.4 & - & - & $\mathrm{LC}$ \\
\hline 368 & Poaceae & Poa pratensis L. & $\mathrm{E} 1.2$ & - & - & $\mathrm{LC}$ \\
\hline 369 & Poaceae & $\begin{array}{l}\text { Polypogon monspeliensis (L.) } \\
\text { Desf. }\end{array}$ & $\mathrm{E} 4.4$ & - & - & $\mathrm{LC}$ \\
\hline 370 & Poaceae & $\begin{array}{l}\text { Puccinellia convoluta } \\
\text { (Hornem.) P. Fourr. }\end{array}$ & E4.4 & - & - & $\mathrm{LC}$ \\
\hline 371 & Poaceae & $\begin{array}{l}\text { Puccinellia koeieana Melderis } \\
\text { subsp. anatolica Kit Tan }\end{array}$ & El.2 & Endemik & - & $\mathrm{LC}$ \\
\hline 372 & Poaceae & Secale montamun Guss. & E4.4 & & & $\mathrm{LC}$ \\
\hline 373 & Poaceae & Stipa capillata L. & $\mathrm{E} 4.4$ & & & $\mathrm{LC}$ \\
\hline 374 & Poaceae & Stipa holosericea Trin & $\mathrm{E} 4.4$ & & Ir.-Tur. ele. & $\mathrm{LC}$ \\
\hline 375 & Poaceae & $\begin{array}{l}\text { Taeniatherum caput-medusae } \\
\text { (L.) Nevslci subsp. crinitum } \\
\text { (Schreber) Melderis }\end{array}$ & 11.3 & & & $\mathrm{LC}$ \\
\hline 377 & Poaceae & Triticum aestivum L. & 11.3 & & & $\mathrm{LC}$ \\
\hline 378 & Poaceae & Zea mays L. & 11.3 & & & $\mathrm{LC}$ \\
\hline 379 & Poaceae & $\begin{array}{l}\text { Zingeria biebersteiniana } \\
\text { (Claus) P. Smirnov subsp. } \\
\text { biebersteiniana }\end{array}$ & E1.2 & & Ir.-Tur. ele. & LC \\
\hline 380 & Typhaceae & Typha latifolia L. & D6.1 & & & $\mathrm{LC}$ \\
\hline
\end{tabular}




\section{CONCLUSION AND DISCUSSION}

The outcomes of this research were based on the examination of 874 plant specimens collected in 2 years, field observations, and collection of literature data on the issue.

As a result of naming of the plant specimens, 237 genera belonging to 65 families, 383 species, 3 subspecies, 2 varieties, as a total of 388 taxons were determined. 52 of the identified 383 species $(13.57 \%)$ were endemic.

The first ten families consisting of maximum number of species among the families which contained the identified 388 taxons were in order of; Asteraceae, Poaceae, Fabaceae, Chenopodiaceae, Lamiaceae, Caryophllaceae, Brassiaceae, Apiaceae, Ranunculaceae, and Scrophulariaceae; and the family spektrum is shown in Figure 2.

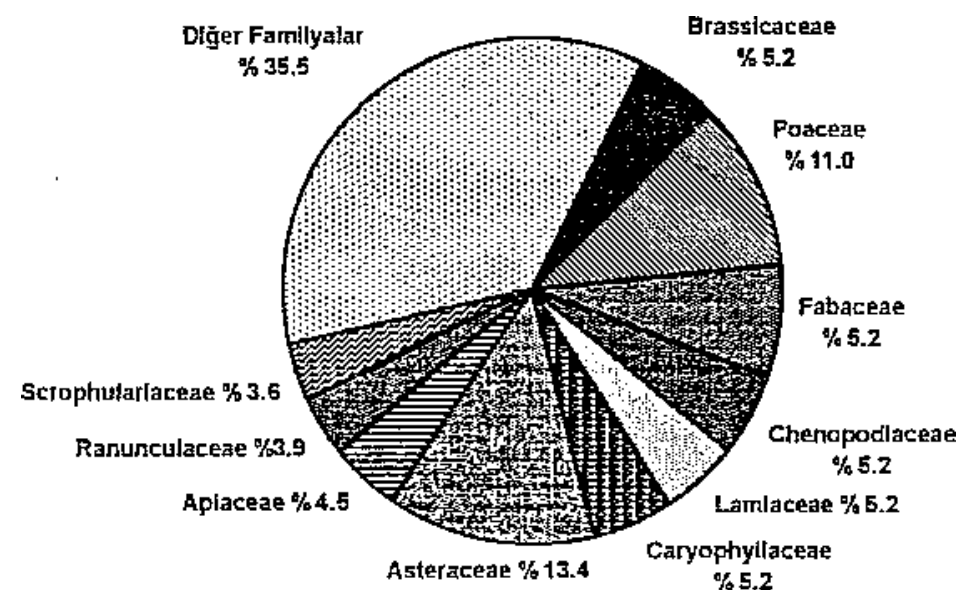

Figure 2. Spectrum of the first ten families consisting of maximum number of species

Diğer familyalar: other families

Distribution spectrum of the species of the research area according to phytogeographic regions is shown in Figure 3. Iranian-Turanian phytogeography region had 102 species, which was the maximum number of species in our area, and the others were respectively, European-Siberian region had 29 species, Mediterranean region had 13 species, cosmopolites had 82 species, and there were 123 species with unknown phytogeographic region (Figure 2).

The research area was situated in the middle of Central Anatolia and it was in Iranian-Turanian floristic region phytogeographically. Various writers indicated different borders for Iranian-Turanian region and used different names. Based on the arguments presented by some writers and collected data, the thought of dividing Iranian-Turanian region into the western and eastern sub-sections draws attention. Central Anatolian region is included in Iranian-Anatolian province, which is located in the western IranianTuranian sub-region of Iranian-Turanian region. 


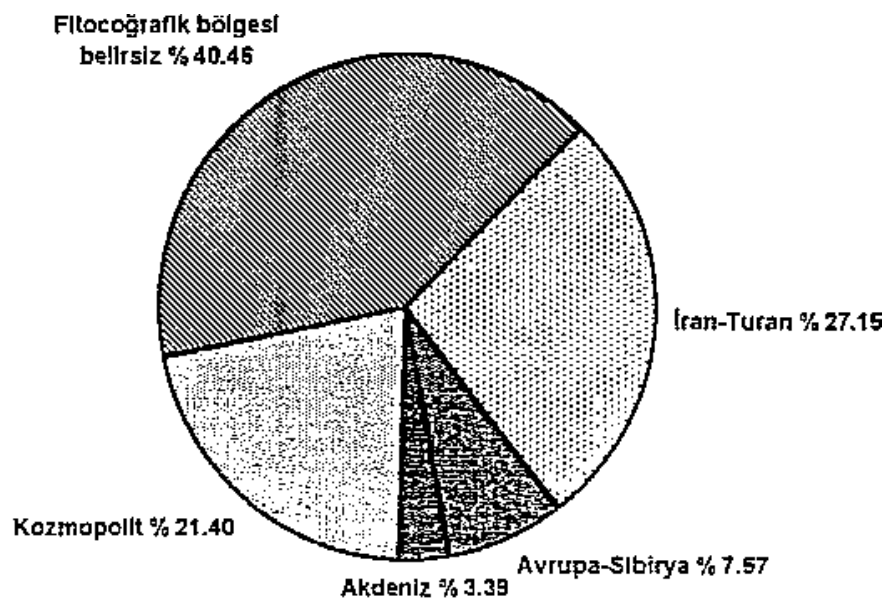

Figure 3. Spectrum of Phytogeographical Region of Species

Fitocoğrafik bölgesi belirsiz: with unknown phytogeographic region / kozmopolit: cosmopolitan / IranianTuranian / European/Syberian / Akdeniz: Mediterranean

Increasing bird population at Lake Seyfe draws attention of many hunters. Hunting of birds by hoaxing them in various ways and with various tools around the Lake to the level of slaugher should not be permitted absolutely. Hunting is prohibited at Seyfe Lake and its vicinity that are designated as a nature reserve area. However, there is no competent authority emerged yet to implement this prohibition.

Lake Seyfe has a rare and extremely sensitive ecosystem and the basin is remarkable for the presence of birds. These aquatic birds have global scale significance and their protection depends on the preservation of primarily the lake ecosystem. Thus, factors disrupting ecosystem of Lake Seyfe must be taken under control and the outdated insight characterizing wetlands as bogs that need to be dried and opened to agriculture must be abandoned.

\section{BIBLIOGRAPHY}

- Akman, Y., Ikklim ve Biyoiklim, Palme Yayın, Ankara, 1990.

- Başbakanlık D.M.İ. Gn.Md. Ortalama, Extrem Sıcaklık ve Yağış Değerleri Bülteni (Günlük Aylık), Türk Tarih Kurumu Matbaası, Ankara, 1984.

- Boissier, E., Flora Orientalis, 1-6, Geneve et Basel, 1867-1888.

- Chapman, V.J., Geohotanical Foundation of the Middle East, I-II, Gustav Fischer Verlag Stutgart, 1973.

• Çırpıcı, A., "Türkiye'nin Flora ve Vejetasyonu Üzerindeki Çalışmalar" Doğa TU. Botanik D. 217 232, 1987.

- Davis, P.H., Flora of Turkey and the East Aegean Islands, 1-10, Edinburgh, 1995-1985.

- DSİ, Kırşehir Seyfe Ovası Hidrojeolojik Etüd Raporu, Devlet Su İşleri, Genel Müdürlüğü, Ankara, 1979.

- Elmacı, A., Obalı, O., "Kırşehir-Seyfe Gölü Bentik Alg Florası." İstanbul Üniv. Su Ürünleri Dergisi, 1:41-64, 1992. 
- Erol, O. "Anadolu'da Kuvatemer Pluviyal Koşullar ve Özellikle Güney İç Anadolu'da Son Buzul Çağından Bugüne Kadar Olan Çevresel Değişmeler", Coğ. Araş. Der. No: 9.5-16, Ankara, 1980.

• Ertan, A., Kılıç, A., Kasparek, M., Türkiye'nin Önemli Kuş Alanları, D.H.K.D. Yayını, 1989.

- Evan, G., Townsend, C.C., Flora of Iraq. Vol.9, Baghdad, 1968.

- Eyioğlu, Y., Malya Devlet Üretme Çiftliği Tuzlu ve Alkali Topraklarının Mekanik Yöntemle Islahı Üzerine Araştırma, Doktora Tezi, Ankara Üniversitesi Ziraat Fakültesi, 1983.

- Eyüboğlu, Ö. Seyfe Gölü (Kırşehir) Tabiatı Koruma AlanınınFlorası, Doktora Tezi, Gazi Üniversitesi Fen Bilimleri Enstitüsü, Ankara, 1995.

• Eyüboğlu, Ö., “Kızılcahamam Soğuksu Milli Parkında Tespit Edilmiş Endemik Bitkilerimiz” Orman Mühendisliği, sayı: 2, 1997.

- Eyüboğlu, Ö., Anadolu'dan (B5) Yeni Florostik Kayıtlar. Orman Mühendisliği, sayı: 3, 1996.

- Gıda Tarım ve Hayvancılık Bakanlığı, D.M.İ., Ortalama ve Extrem Klymetler Meteoroloji Bülteni, Başbakanlık Basımevi, 1974.

- Gülpınar, T., Türkiye'nin Uluslararası Öneme Sahip Sulak Sahaları, (Yayınlanmamış Rapor).

- Gürpınar, T., Kuş Göçü Açısından Türkiye'nin Önemi, Türkiye ve Balkan Ülkelerinde Yaban Hayatı Uluslararası Sempozyumu, 1987.

- Heywood, V.H., Tutin, G.T. (ed.), Flora Europeae, Vol: I-V, Cambridge Univ. Press (19641981).

- Hutchinson, J., The Genera of Flowering Plants (Angiospermae), Dicotyledones, Vol. 1, Oxford, 1964.

- Köy İşleri ve Kooperatifleri Bakanlığı, Kızılırmak Havzası Toprakları, T.S.İ. Gn.Md. Yayınlan: 286, Havza No: 15, Raporlar Serisi: 71, 1970.

- Munsuz, Malya Devlet Üretme Çiftliği Ön Çalışma Raporu, Tarım Bakanlı̆̆l, 1959.

- Özer, A.Ö., Sustainable Development Strategy in Regional Planning and A Case Study on Seyfe Region, A Master's Thesis in Regional Planning METU, ANKARA, 1990.

- Ramsar Convention, Convention on Wetlands of International Importance Especially as Waterfowl Habitat, Held at Ramsar, Iran 30 January-3 February, 1971.

- Ünver, I., Seyfe Havzası Ekolojisinin Korunması ve Islahı Konusunda Alternatif Bir Çözüm Önerisi, A.Ü. Ziraat Fakültesi, 1990.

- Yiğitbaşaoğlu, H., Seyfe Gölü'nün Jeolojisi, Doktora Tezi, Dil Tarih Coğrafya Fakültesi, Ankara.

- Yurdakulol, E., Ercoşkun, T., "Orta Anadolu'da Çorak Alanların Vejetasyonu Üzerinde Ekolojik ve Sintaksonomik Araştırma" TÜBİTAK Doğa-Tr. J. of Botany, 14 : 109-123, 1990. 